\title{
Fauna of the Ladybird Beetles (Coleoptera: Coccinellidae) and their Associated Host Plants from Southern Syria
}

\author{
Nazir Khalil ${ }^{1}$, Abdoulrahman Mourad ${ }^{2}$, Mahmoud Karoum ${ }^{3}$, Mohammad Abu Baker ${ }^{4}$ \\ and Zuhair Amr ${ }^{5, *}$ \\ ${ }^{1}$ Al-Furat University, Sciences Faculty, Al-Raqa, Syria $;^{2}$ Damascus University, Sciences Faculty, Biology Animal Department, Damascus, \\ Syria; $;^{3}$ Aleppo University, Sciences Faculty, Biology Animal Department, Aleppo, Syria; $;^{4}$ Department of Biology, The University of Jordan, \\ Amman, Jordan; ${ }^{5}$ Department of Biology, Jordan University of Sciences and Technology, Irbid, Jordan.
}

Received: November 4, 2020; Revised: December 27, 2020; Accepted: January 28, 2021

\begin{abstract}
The coccinellids of southern Syria were studied based on 7418 specimens collected during 2001 to 2005. Fifty-one species and subspecies belonging to 21 genera of 11 tribes and two subfamilies (Microweiseinae and Coccinellinae) were recorded. Fifteen species are recorded for the first time in Syria. Host plants species for each coccinellid species were recorded.
\end{abstract}

Keywords: Coccinellids, Coccinellidae, Coleoptera, systematics, Syria, host plant.

\section{Introduction}

Ladybird beetles (Coccinellidae) constitute one of the largest families of coleopterous insects, including over 6000 species (Hodek et al., 2015)). The overwhelming majority of lady beetles are predators, feeding of aphids, psyllids, whiteflies, mealybugs, scales, thrips, spider mites, leaf beetle larvae and other small arthropods (Kuznetsov, 1997).

The species of family Coccinellidae in the Middle East, with 61 species in Iraq (Ali et al., 1990), 142 species in Iran (Biranvand et al., 2016; Mesbah et al., 2016), 16 species in Jordan (Alawi, 1989), 71 species in Palestine (Halperin et al., 1995; Najajrah et al., 2019), 35 species in Saudi Arabia (Fürsch, 1979; Raimundo and van Harten, 2000), 84 species in Turkey (Yurtsever 2001), 22 species in the United Arab Emirates (Raimundo et al., 2008) and 73 species in Yemen (Raimundo and van Harten, 2000). Prior to this study, only 11 species had been recorded from southern Syria without sufficient data on their distribution or ecology (Winkler, 1927; El-Hariri, 1968 and 1971; Kabakibi, 1993; Almatni et al., 1999; Bascheer and Abo Alshamat 2004; Almatni and Khalil, 2008).

The aim of the present work is to study the biodiversity and distribution of the coccinellid beetles in southern Syria, with notes on their host plants.

\section{Materials and methods}

Sampling was conducted in southern Syria covering five Governorates (Damascus, Damascus Rural, AlQuneitra, Dar`a and As`Sweida) located at different altitudes (280-2400m asl. (Figure 1, Table 1). A total of 7418 adult ladybirds was collected from agricultural and non-agricultural areas during three years (August 2001July 2003), and other specimens were collected during 2004 and 2005.

Ladybird beetles were collected from branches and foliage of trees or herbaceous plants. Also, trees were shaken and insects were collected in a cloth tray with an insect net or by an aspirator. Each specimen was labeled with data about host plants, locality, and date. Specimens were preserved in $70 \%$ ethanol, and other specimens were dry mounted and preserved in collection boxes. Whole speciemns were photographed by Canon camera, while gential structure were extracted (Tegmen and penis, for the males and the spermatheca for the females), boiled in $10 \%$ $\mathrm{KOH}$, washed in water , different concentrations of alcohol $70 \%$, $95 \%$ and $100 \%$, and xylene, then mounted on slides and drawn using camera lucida. Specimens are deposited at the Department of Animal Biology, Damascus University.

\footnotetext{
Corresponding author e-mail: amrz@just.edu.jo.
} 


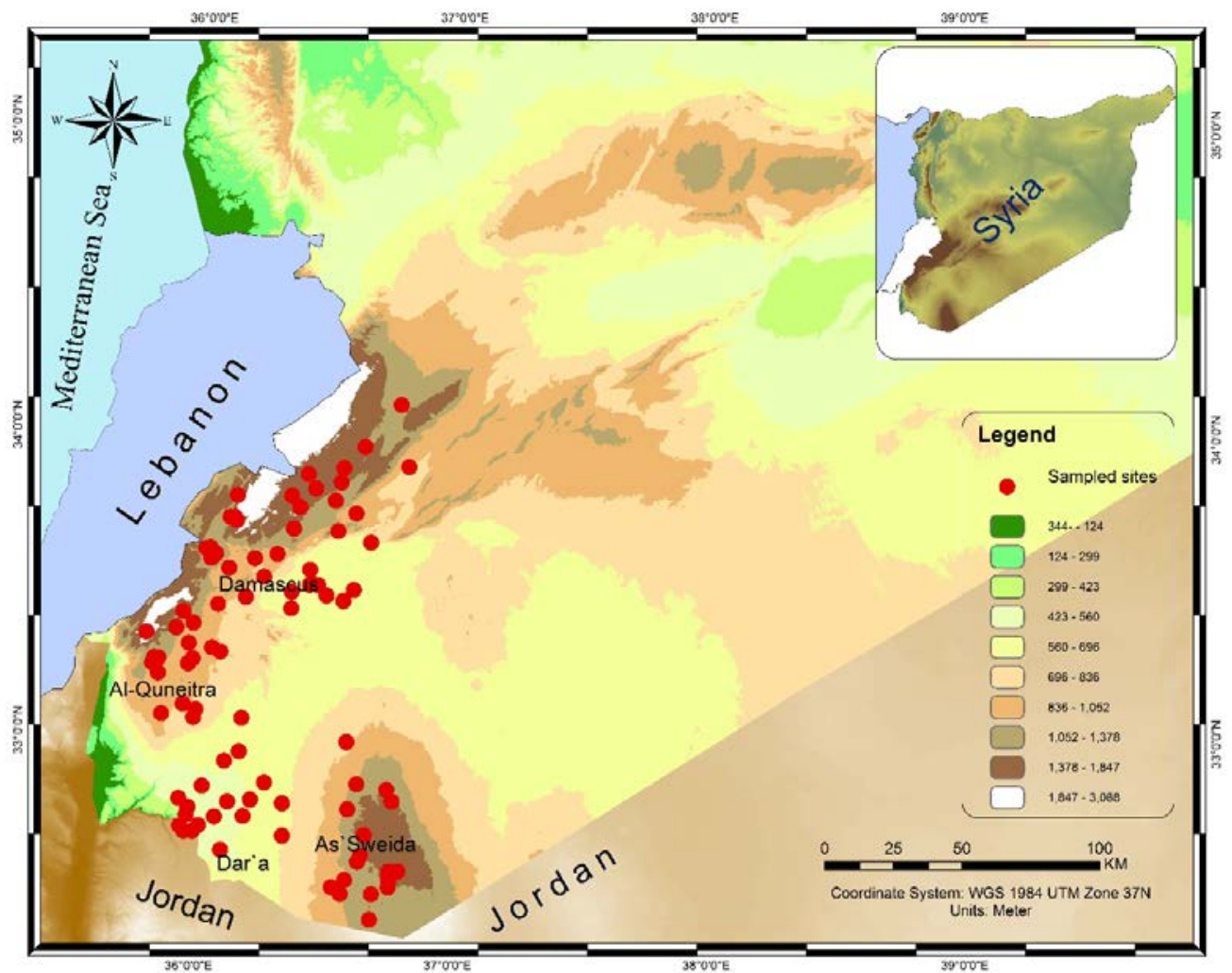

Figure 1: Collecting localities with their coordinates.

Species identification was carried out using different taxonomic keys and taxonomic references (Dauguet, 1949; Chapin, 1965; Fürsch, 1967a; 1979, 1989; IablokoffKhnzorian, 1971, 1982; Hodek, 1973; Gourreau, 1974; Chazeau et al., 1974; Leeper 1975; Uygun 1981, Uygun and Fürsch, 1981; Canepari, 1983; Canepari et al., 1985; Klausnitzer and Klausnitzer, 1986; Raimundo and Alves, 1986; Majerus and Kearns, 1989; Duverger, 1991; Kuznetsov, 1997; Raimundo and van Harten, 2000; Le Monnier and Livory, 2003). Identification of most species was confirmed by Prof. Dr. Helmut Fürsch (Passau University, Germany).

Table 1: Collecting localities with their coordinates.

\begin{tabular}{|c|c|c|c|c|c|}
\hline localities & Latitude & Longitude & Localities & Latitude & Longitude \\
\hline Airport Street & $33^{\circ} 28^{\prime} 36^{\prime \prime}$ & $36^{\circ} 21^{\prime} 48^{\prime \prime}$ & Kulaiaa & $33^{\circ} 17^{\prime} 12^{\prime \prime}$ & $36^{\circ} 03^{\prime} 30^{\prime \prime}$ \\
\hline Ahmadia & $33^{\circ} 28^{\prime}$ & $36^{\circ} 30^{\prime}$ & Maalula & $33^{\circ} 50^{\prime} 21^{\prime \prime}$ & $36^{\circ} 32^{\prime} 57^{\prime \prime}$ \\
\hline Ajami & $32^{\circ} 42^{\prime}$ & $35^{\circ} 57^{\prime}$ & Ma`adamiyeh Al-sham & $33^{\circ} 27^{\prime} 21^{\prime \prime}$ & $36^{\circ} 11^{\prime} 06^{\prime \prime}$ \\
\hline Addimass & $33^{\circ} 33^{\prime}$ & $36^{\circ} 07^{\prime}$ & Maissalon & $33^{\circ} 35^{\prime} 44^{\prime \prime}$ & $36^{\circ} 03^{\prime} 49^{\prime \prime}$ \\
\hline Al-Dumayr & $33^{\circ} 38^{\prime} 37^{\prime \prime}$ & $36^{\circ} 40^{\prime} 11^{\prime \prime}$ & Masehara & $33^{\circ} 06^{\prime}$ & $35^{\circ} 57^{\prime}$ \\
\hline Al-Harra & $33^{\circ} 03^{\prime} 24^{\prime \prime}$ & $35^{\circ} 59^{\prime} 33^{\prime \prime}$ & Mashkok & $32^{\circ} 24^{\prime} 23^{\prime \prime}$ & $36^{\circ} 41^{\prime} 37^{\prime \prime}$ \\
\hline Al-Hameh & $33^{\circ} 35^{\prime}$ & $36^{\circ} 13^{\prime}$ & Mugr Almeer & $33^{\circ} 18^{\prime}$ & $35^{\circ} 58^{\prime}$ \\
\hline Al-Kafr & $32^{\circ} 36^{\prime} 56^{\prime \prime}$ & $36^{\circ} 39^{\prime} 16^{\prime \prime}$ & Mzireeb & $32^{\circ} 42^{\prime} 13^{\prime \prime}$ & $36^{\circ} 01^{\prime} 21^{\prime \prime}$ \\
\hline Alqraya & $32^{\circ} 32^{\prime} 06^{\prime \prime}$ & $36^{\circ} 35^{\prime} 39^{\prime \prime}$ & Nahaj & $32^{\circ} 41^{\prime}$ & $36^{\circ} 00^{\prime}$ \\
\hline Al-Qutayfeh & $33^{\circ} 44^{\prime} 17^{\prime \prime}$ & $36^{\circ} 36^{\prime} 35^{\prime \prime}$ & Nahta & $32^{\circ} 46^{\prime} 56^{\prime \prime}$ & $36^{\circ} 20^{\prime} 46^{\prime \prime}$ \\
\hline Annashabyeh & $33^{\circ} 30^{\prime}$ & $36^{\circ} 28^{\prime}$ & Namer & $32^{\circ} 47^{\prime} 28^{\prime \prime}$ & $36^{\circ} 13^{\prime} 18^{\prime \prime}$ \\
\hline Ashiphonieh & $33^{\circ} 33^{\prime}$ & $36^{\circ} 26^{\prime}$ & Nasseria & $33^{\circ} 52^{\prime} 99^{\prime \prime}$ & $36^{\circ} 48^{\prime} 49^{\prime \prime}$ \\
\hline Attall & $33^{\circ} 35^{\prime} 55^{\prime \prime}$ & $36^{\circ} 18^{\prime} 15^{\prime \prime}$ & Ophania & $33^{\circ} 12^{\prime}$ & $35^{\circ} 51^{\prime}$ \\
\hline Attawani & $33^{\circ} 46^{\prime} 43^{\prime \prime}$ & $36^{\circ} 30^{\prime} 99^{\prime \prime}$ & Orman & $32^{\circ} 30^{\prime} 49^{\prime \prime}$ & $36^{\circ} 45^{\prime} 49^{\prime \prime}$ \\
\hline Assaida Zainab & $33^{\circ} 25^{\prime} 19^{\prime \prime}$ & $36^{\circ} 21^{\prime} 51^{\prime \prime}$ & Orman Mountain & $32^{\circ} 31^{\prime} 54^{\prime \prime}$ & $36^{\circ} 45^{\prime} 54^{\prime \prime}$ \\
\hline Assal Alwared & $33^{\circ} 51^{\prime} 48^{\prime \prime}$ & $36^{\circ} 25^{\prime} 10^{\prime \prime}$ & Qanawat & $32^{\circ} 46^{\prime}$ & $36^{\circ} 36^{\prime}$ \\
\hline
\end{tabular}

o assess the occurrence and preference of coccinellid species on plant hosts, we measured species richness (total number of species recorded), species incidence (the number of plant host at which a species occurred). The number of coccinellid species was plotted against the number of plant hosts to distinguish specialist (coccinellids occurring on less than 10 plant hosts) vs. generalist species (those occurring on over 30 plant hosts). To assess the preferred plant hosts (plants on which more than 15 coccinellid occurred) the number of plant hosts was plotted against the total number of coccinellids. 
Table 1. cont

\begin{tabular}{|c|c|c|c|c|c|}
\hline Autaiba & $33^{\circ} 29^{\prime} 12^{\prime \prime}$ & $36^{\circ} 36^{\prime} 22^{\prime \prime}$ & Qatana & $33^{\circ} 25^{\prime} 50^{\prime \prime}$ & $36^{\circ} 04^{\prime} 38^{\prime \prime}$ \\
\hline Azzabadani & $33^{\circ} 42^{\prime} 26^{\prime \prime}$ & $36^{\circ} 08^{\prime} 36^{\prime \prime}$ & Rankus & $33^{\circ} 45^{\prime} 16^{\prime \prime}$ & $36^{\circ} 23^{\prime} 23^{\prime \prime}$ \\
\hline Baka & $32^{\circ} 29^{\prime} 22^{\prime \prime}$ & $36^{\circ} 34^{\prime} 42^{\prime \prime}$ & Rankus Mountain & $33^{\circ} 47^{\prime} 30^{\prime \prime}$ & $36^{\circ} 21^{\prime} 25^{\prime \prime}$ \\
\hline Barad & $32^{\circ} 30^{\prime} 38^{\prime \prime}$ & $36^{\circ} 32^{\prime} 36^{\prime \prime}$ & Sabsaba & $33^{\circ} 05^{\prime}$ & $36^{\circ} 00^{\prime}$ \\
\hline Beqaasem & $33^{\circ} 24^{\prime} 14^{\prime \prime}$ & $35^{\circ} 56^{\prime} 37^{\prime \prime}$ & Sad alaain & $32^{\circ} 35^{\prime} 43^{\prime \prime}$ & $36^{\circ} 38^{\prime} 34^{\prime \prime}$ \\
\hline Beer Ajam & $33^{\circ} 04^{\prime}$ & $35^{\circ} 52^{\prime}$ & Sahm al-joulan & $32^{\circ} 47^{\prime} 24^{\prime \prime}$ & $35^{\circ} 56^{\prime} 35^{\prime \prime}$ \\
\hline Beet jen- Mazraa & $33^{\circ} 15^{\prime}$ & $35^{\circ} 51^{\prime}$ & Salkhad & $32^{\circ} 29^{\prime} 24^{\prime \prime}$ & $36^{\circ} 41^{\prime} 59^{\prime \prime}$ \\
\hline Bludan & $33^{\circ} 43^{\prime}$ & $36^{\circ} 07^{\prime}$ & Sanameen & $33^{\circ} 03^{\prime} 32^{\prime \prime}$ & $36^{\circ} 10^{\prime} 48^{\prime \prime}$ \\
\hline Bludan Mountain & $33^{\circ} 43^{\prime}$ & $36^{\circ} 08^{\prime}$ & Serghaya & $33^{\circ} 47^{\prime} 21^{\prime \prime}$ & $36^{\circ} 08^{\prime} 37^{\prime \prime}$ \\
\hline Damascus (Qasioun) & $33^{\circ} 31^{\prime} 23^{\prime \prime}$ & $36^{\circ} 15^{\prime} 15^{\prime \prime}$ & Sarkha & $33^{\circ} 53^{\prime} 10^{\prime \prime}$ & $36^{\circ} 33^{\prime} 31^{\prime \prime}$ \\
\hline Dar`a & $32^{\circ} 36^{\prime} 90^{\prime \prime}$ & $36^{\circ} 06^{\prime} 40^{\prime \prime}$ & Sasaa & $33^{\circ} 15^{\prime}$ & $35^{\circ} 59^{\prime}$ \\
\hline Deir Al-ashairr & $33^{\circ} 34^{\prime} 49^{\prime \prime}$ & $36^{\circ} 02^{\prime} 44^{\prime \prime}$ & Saura & $32^{\circ} 59^{\prime} 16^{\prime \prime}$ & $36^{\circ} 35^{\prime} 27^{\prime \prime}$ \\
\hline Deir Atyeh & $34^{\circ} 05^{\prime} 48^{\prime \prime}$ & $36^{\circ} 46^{\prime} 42^{\prime \prime}$ & Sehaileia & $32^{\circ} 55^{\prime}$ & $36^{\circ} 07^{\prime}$ \\
\hline Dorin & $33^{\circ} 14^{\prime}$ & $35^{\circ} 58^{\prime}$ & Shahba & $32^{\circ} 51^{\prime}$ & $36^{\circ} 38^{\prime}$ \\
\hline Durbol & $33^{\circ} 21^{\prime}$ & $35^{\circ} 55^{\prime}$ & Sheikh Saad & $32^{\circ} 50^{\prime}$ & $36^{\circ} 02^{\prime}$ \\
\hline Ein alarab & $32^{\circ} 41^{\prime}$ & $36^{\circ} 40^{\prime}$ & Sheikh Miskin & $32^{\circ} 47^{\prime}$ & $36^{\circ} 08^{\prime}$ \\
\hline Erneh & $33^{\circ} 20^{\prime}$ & $35^{\circ} 48^{\prime}$ & Seydnaya & $33^{\circ} 41^{\prime}$ & $36^{\circ} 22^{\prime}$ \\
\hline Fakiia & $32^{\circ} 56^{\prime} 55^{\prime \prime}$ & $36^{\circ} 10^{\prime} 24^{\prime \prime}$ & Southern Al-Qutayfeh & $33^{\circ} 40^{\prime} 44^{\prime \prime}$ & $36^{\circ} 32^{\prime} 27^{\prime \prime}$ \\
\hline Ghoujygoujyat Mont. & $32^{\circ} 32^{\prime} 57^{\prime \prime}$ & $36^{\circ} 45^{\prime} 51^{\prime \prime}$ & Taamri Mountain & $32^{\circ} 33^{\prime} 54^{\prime \prime}$ & $36^{\circ} 45^{\prime} 52^{\prime \prime}$ \\
\hline Hadar & $33^{\circ} 15^{\prime}$ & $35^{\circ} 50^{\prime}$ & Tafas & $32^{\circ} 44^{\prime}$ & $36^{\circ} 05^{\prime}$ \\
\hline Haran Al-awameed & $33^{\circ} 27^{\prime}$ & $36^{\circ} 34^{\prime}$ & Taima & $32^{\circ} 50^{\prime}$ & $36^{\circ} 45^{\prime}$ \\
\hline Houch arab & $33^{\circ} 49^{\prime}$ & $36^{\circ} 27^{\prime}$ & Tal Shihab & $32^{\circ} 41^{\prime}$ & $35^{\circ} 58^{\prime}$ \\
\hline Izraa & $32^{\circ} 50^{\prime} 56^{\prime \prime}$ & $36^{\circ} 15^{\prime} 89^{\prime \prime}$ & Tal-loz & $32^{\circ} 34^{\prime}$ & $36^{\circ} 48^{\prime}$ \\
\hline Jeelin & $32^{\circ} 45^{\prime} 45^{\prime \prime}$ & $35^{\circ} 58^{\prime} 46^{\prime \prime}$ & Tarba & $32^{\circ} 47^{\prime} 44^{\prime \prime}$ & $36^{\circ} 46^{\prime} 19^{\prime \prime}$ \\
\hline Jeesrin & $33^{\circ} 30^{\prime}$ & $36^{\circ} 26^{\prime}$ & Trounja & $33^{\circ} 14^{\prime} 40^{\prime \prime}$ & $35^{\circ} 50^{\prime} 48^{\prime \prime}$ \\
\hline Jubata Al-Khashab & $33^{\circ} 13^{\prime} 56^{\prime \prime}$ & $35^{\circ} 49^{\prime} 36^{\prime \prime}$ & Wadi Al-Qaren & $33^{\circ} 36^{\prime} 44^{\prime \prime}$ & $36^{\circ} 01^{\prime} 31^{\prime \prime}$ \\
\hline Kafr- Hawar & $33^{\circ} 22^{\prime}$ & $35^{\circ} 59^{\prime}$ & Wadi Jeelin & $32^{\circ} 44^{\prime} 13^{\prime \prime}$ & $35^{\circ} 58^{\prime} 27^{\prime \prime}$ \\
\hline Kanaker & $33^{\circ} 16^{\prime} 28^{\prime \prime}$ & $36^{\circ} 05^{\prime} 28^{\prime \prime}$ & Wadi Shihab & $32^{\circ} 41^{\prime}$ & $35^{\circ} 58^{\prime}$ \\
\hline Karak & $32^{\circ} 40^{\prime} 31^{\prime \prime}$ & $36^{\circ} 20^{\prime} 57^{\prime \prime}$ & Yabrud & $33^{\circ} 57^{\prime} 24^{\prime \prime}$ & $36^{\circ} 38^{\prime} 23^{\prime \prime}$ \\
\hline Kharabo & $33^{\circ} 30^{\prime} 08^{\prime \prime}$ & $36^{\circ} 27^{\prime} 19^{\prime \prime}$ & Zarzar & $33^{\circ} 36^{\prime} 54^{\prime \prime}$ & $36^{\circ} 02^{\prime} 30^{\prime \prime}$ \\
\hline Kherbeet Gazaleh & $32^{\circ} 44^{\prime} 13^{\prime \prime}$ & $36^{\circ} 11^{\prime} 41^{\prime \prime}$ & Zeizon & $32^{\circ} 42^{\prime}$ & $35^{\circ} 57^{\prime}$ \\
\hline
\end{tabular}

\section{Results}

Fifty-one species and subspecies belonging to 21 genera representing 11 tribes within two subfamilies were recorded from southern Syria. Fifteen species are new records to Syria (Table 2). Higher taxonomic classification and scientific names were followed according to those in Kováŕ (2007), Ślipiński (2007), Seago et al. (2011) and Robertson et al. (2015).

Table 2. List of coccinellid species in Southern Syria.

\begin{tabular}{lll}
\hline Species & $\begin{array}{l}\text { No. of } \\
\text { sites }\end{array}$ & $\begin{array}{l}\text { Total No. } \\
\text { collected } \\
\text { speciemns }\end{array}$ \\
\hline $\begin{array}{l}\text { Subfamily Microweiseinae } \\
\text { Tribe Microweiseini }\end{array}$ & & \\
$\begin{array}{l}\text { Pharoscymnus ovoideus Sicard, 1929 } \\
\text { Pharoscymnus setulosus (Chevrolat, 1861)* } \\
\text { Subfamily Coccinellinae }\end{array}$ & 1 & 272 \\
$\begin{array}{l}\text { Tribe Coccidulini } \\
\text { Cryptolaemus montrouzieri Mulsant, 1853 }\end{array}$ & 3 & 6 \\
Lindorus lophantae (Blaisdéll, 1892)* & 4 & 80 \\
\hline
\end{tabular}

\begin{tabular}{|c|c|c|}
\hline \multicolumn{3}{|l|}{ Tribe Diomini } \\
\hline $\begin{array}{l}\text { Diomus rubidus (Motschulsky, 1837) } \\
\text { Tribe Hyperaspidini }\end{array}$ & 1 & 1 \\
\hline $\begin{array}{l}\text { Hyperaspis (Hyperaspis) femorata } \\
\text { (Motschulsky, 1837)* }\end{array}$ & 1 & 2 \\
\hline $\begin{array}{l}\text { Hyperaspis (Hyperaspis) histeroides } \\
\text { (Faldermann, 1837)* }\end{array}$ & 8 & 12 \\
\hline $\begin{array}{l}\text { Hyperaspis (Hyperaspis) syriaca Weise, } \\
1885\end{array}$ & 1 & 16 \\
\hline \multicolumn{3}{|l|}{ Tibe Scymnini } \\
\hline Clitostethus arcuatus (Rossi, 1794) & 3 & 40 \\
\hline $\begin{array}{l}\text { Nephus (Bipunctatus) bipunctatus } \\
\text { (Kugelann, 1794)* }\end{array}$ & 32 & 97 \\
\hline $\begin{array}{l}\text { Nephus (Bipunctatus) includens (Kirsch, } \\
\text { 1870) }\end{array}$ & 2 & 2 \\
\hline Nephus (Nephus) ludyi (Weise, 1879)* & 11 & 115 \\
\hline Nephus (Nephus) merkli Fürsch, 1994* & 8 & 115 \\
\hline $\begin{array}{l}\text { Nephus (Nephus) quadrimaculatus Herbst, } \\
1783\end{array}$ & 29 & 265 \\
\hline Nephus (Sidis) caucasicus Weise, 1929* & 1 & 1 \\
\hline Nephus (Sidis) hiekei Fürsch, 1965* & 2 & 2 \\
\hline $\begin{array}{l}\text { Nephus (Sidis) kreissli Fürsch \& Uygun, } \\
\text { 1980* }\end{array}$ & 4 & 27 \\
\hline $\begin{array}{l}\text { Scymnus (Mimopullus) pharaonis } \\
\text { Motschulsky, } 1851\end{array}$ & 39 & 317 \\
\hline $\begin{array}{l}\text { Scymnus (Mimopullus) flagellisiphonatus } \\
\text { Fürsch, } 1969\end{array}$ & 17 & 26 \\
\hline Scymnus (Mimopullus) flavicollis & 26 & 416 \\
\hline
\end{tabular}


Scymnus (Mimopullus) marinus Mulsant, $\quad 11 \quad 109$ 1850

\begin{tabular}{|c|c|c|}
\hline $\begin{array}{l}\text { Scymnus (Parapullus) abietis (Paykull, } \\
\text { 1798)* }\end{array}$ & 2 & $2+$ \\
\hline Scymnus (Pullus) apetzi Mulsant, 1846* & 34 & 403 \\
\hline Scymnus (Pullus) auritus Thunberg, 1795* & 4 & 5 \\
\hline Scymnus (Pullus) fraxini Mulsant, 1850* & 2 & 62 \\
\hline Scymnus (Pullus) subvillosus (Goeze, 1777) & 30 & 316 \\
\hline Scymnus (Pullus) syriacus (Marseul, 1868) & 28 & 250 \\
\hline $\begin{array}{l}\text { Scymnus (Scymnus) bivulnerus Capra \& } \\
\text { Fürsch, } 1967\end{array}$ & 16 & 52 \\
\hline $\begin{array}{l}\text { Scymnus (Scymnus) frontalis (Fabricius, } \\
1787 \text { ) }\end{array}$ & 2 & 60 \\
\hline $\begin{array}{l}\text { Scymnus (Scymnus) interruptus (Goeze, } \\
1777 \text { ) }\end{array}$ & 13 & 111 \\
\hline Scymnus (Scymnus) nubilus Mulsant, 1850 & 31 & 279 \\
\hline \multicolumn{3}{|l|}{ Tribe Stethorini } \\
\hline $\begin{array}{l}\text { Stethorus (Stethorus) gilvifrons (Mulsant, } \\
\text { 1850) }\end{array}$ & 48 & 1368 \\
\hline \multicolumn{3}{|l|}{ Tribe Chilocorini } \\
\hline Chilocorus bipustulatus (Linnaeus, 1758) & 7 & 42 \\
\hline Exochomus octosignatus (Gebler, 1830)* & 1 & 1 \\
\hline $\begin{array}{l}\text { Exochomus quadripustulatus (Linnaeus, } \\
\text { 1758) }\end{array}$ & 4 & 54 \\
\hline Exochomus undulatus (Weise, 1878) & 2 & 2 \\
\hline $\begin{array}{l}\text { Parexochomus nigromaculatus (Goeze, } \\
\text { 1777) }\end{array}$ & 10 & 24 \\
\hline Parexochomus pubescens (Küster, 1848) & 4 & 6 \\
\hline \multicolumn{3}{|l|}{ Tribe Platynaspidini } \\
\hline Platynaspis luteorubra (Goeze, 1777) * & 8 & 14 \\
\hline \multicolumn{3}{|l|}{ Tribe Noviini } \\
\hline Novius cardinalis (Mulsant, 1850) & 4 & 16 \\
\hline \multicolumn{3}{|l|}{ Tribe Tytthaspidini } \\
\hline $\begin{array}{l}\text { Anisosticta novemdecimpunctata (Linnaeus, } \\
1758)^{*}\end{array}$ & 1 & 1 \\
\hline \multicolumn{3}{|l|}{ Tribe Coccinellini } \\
\hline Adalia (Adalia) bipunctata (Linnaeus, 1758) & 6 & 61 \\
\hline $\begin{array}{l}\text { Adalia (Adalia) decempunctata (Linnaeus, } \\
\text { 1758) }\end{array}$ & 13 & 37 \\
\hline $\begin{array}{l}\text { Coccinella (Coccinella) septempunctata } \\
\text { Linnaeus, } 1758\end{array}$ & 39 & 330 \\
\hline $\begin{array}{l}\text { Coccinella (Spilota) undecimpunctata } \\
\text { aegyptica Reiche, } 1861\end{array}$ & 40 & 152 \\
\hline $\begin{array}{l}\text { Harmonia quadripunctata (Pontoppidan, } \\
\text { 1763) }\end{array}$ & 10 & 59 \\
\hline $\begin{array}{l}\text { Hippodamia (Adonia) variegata (Goeze, } \\
\text { 1777) }\end{array}$ & 56 & 1042 \\
\hline Oenopia conglobata (Linnaeus, 1758) & 41 & 618 \\
\hline Oenopia oncina (Olivier, 1808)* & 5 & 6 \\
\hline $\begin{array}{l}\text { Propylea quatuordecimpunctata (Linnaeus, } \\
\text { 1758) }\end{array}$ & 8 & 121 \\
\hline $\begin{array}{l}\text { Psyllobora (Thea) vigintiduopunctata } \\
\text { (Linnaeus, 1758) }\end{array}$ & 1 & 2 \\
\hline Total & 94 & 7418 \\
\hline
\end{tabular}

Family Coccinellidae Latreille, 1807

Subfamily Microweiseinae Leng, 1920

Tribe Microweiseini Leng, 1920

Pharoscymnus ovoideus Sicard, 1929 (Figs. 2A and 3A)

Material examined (272): Damascus Rural: Attall, (1), 26.9.2001; (1), 8.5.2002; (1), 26.6.2002; (2), 5.9.2002. Wadi Al-Qaren, (4), 19.8.2001; (1), 19.9.2001; (7), 5.9.2002. Yabrud, (2), 25.10.2001; (1), 8.8.2002; (3), 11.9.2002. Sasaa, (5), 13.8.2001; (4), 3.10.2001; (1), 20.3.2002; (5), 2.5.2002; (4), 31.7.2002; (3), 12.9.2002.
Kulaiaa, (1), 7.8.2002. Beqaasem, (2), 21.9.2001; (6), 11.7.2002; (1), 29.8.2002. Mugr Almeer, (1), 26.8.2003. Addimass, (4), 5.9.2002; (9), 17.9.2002; (6), 30.4.2003. Al Hameh, (1), 14.6.2003. Sarkha, (1), 26.9.2001; (4), 11.9.2002; (1), 21.5.2003. Seydnaya, 11: (9), 2.8.2001; (2), 21.5.2003. Al-Qutayfeh, (8), 8.8.2002. Annashabyeh, (4), 28.8.2001; (2), 21.11.2001; (5), 24.7.2002; (2), 27.7.2002. Kharabo, (3), 22.8.2001; (1), 26.9.2001; (1), 25.10.2001; (1), 23.5.2002; (1), 11.7.2002; (2), 25.5.2003; (4), 2.7.2003. Ma`adamiyeh Al-sham, (1), 7.10.2001; (2), 6.12.2001; (1), 6.3.2002; (1), 12.4.2002; (16), 30.8.2002; (3), 9.9.2002; (15), 4.10.2002; (1), 1.11.2002; (1), 4.12.2002; (1), 16.1.2003; (1), 13.3.2003; (1), 6.7.2003; (2), 11.7.2003; (2), 23.7.2003; (1), 27.7.2003; (5), 9.8.2003; (3), 13.8.2003. Damascus: Qasioun, (3), 13.5.2003. Al-Quneitra: Beer Ajam, (1), 20.3.2002; (2), 12.9.2002; (1), 2.10.2002; (1), 19.3.2003. Jubata AlKhashab, (1), 10.4.2002; (4), 16.5.2002; (2), 11.7.2002; (3), 29.8.2002; (2), 26.8.2003. Hadar, (6), 31.7.2002. Trounja, (1), 29.8.2002. Ophania, (1), 10.9.2001. As 'Sweida: Al-Kafr, (1), 11.5.2003. Ein alarab 12: (2), 7.8.2001; (2), 29.8.2001; (1), 21.8.2002; (6), 23.10.2002; (1), 2.6.2003. Orman mountain, (1), 27.9.2001. Orman, (1), 21.8.2002. Qanawat, (1), 20.9.2001; (2), 11.4.2002. Taima, (12), 11.9.2001. Mashkok, (1), 20.9.2001. Alqrayya (2), 20.9.2001. Dar`a: Jeelin 4: (2), 30.8.2001; (1), 24.10.2001; (1), 3.7.2002. Wadi Jeelin, (1), 25.4.2002; (1), 22.8.2002. Zeizon, (3), 2.10.2002; (1), 13.11.2002; (2), 23.4.2003. Ajami (1), 18.7.2002. Kherbeet Gazaleh, (1), 7.2.2002. Nahaj, (1), 24.10.2001. Sehaileia, (2), 26.9.2001. Fakiia, (1), 28.3.2002. Al-Harra, (4), 4.9.2001; (4), 2.10.2002; (1), 13.11.2002.

Remarks: It was recorded from Egypt, Jordan, Palestine and Syria (Alfieri, 1976; Halperin et al., 1995; Kováŕ, 2007).

Host species: Amygdalus sp., Cedrus libani, Citrus sp., Crataegus sp., Cupressus sp., Ficus carica, Juglans regia, Medicago sativa, Morus alba, Quercus calliprinos, Olea europaea, Phaseolus vulgaris, Pinus sp., Pistacia vera, Punica granatum, Prunus armeniaca, Prunus persica, Pyrus communis, Schinus molle, Solanum melongena, Zea mays, and thistle plants.

Pharoscymnus setulosus (Chevrolat, 1861) (Figs. 2B and 3B)

Material examined (1): Damascus Rural: Southern Al-Qutayfeh, (1), 15.8.2001.

Remarks: Known from Spain, North Africa, the Middle East, and Yemen (Raimundo and van Harten, 2000, Kovár, 2007). This species is recorded for the first time in Syria.

Host species: Pinus sp.

\section{Subfamily Coccinellinae Latreille, 1807}

Tribe Coccidulini Mulsant, 1846

Cryptolaemus montrouzieri Mulsant, 1853 (Figs. 2C and 3C).

Material examined (6): Dar`a: Wadi Jeelin (1), 24.10.2001; (1), 28.3.2002. Zeizon, (1), 24.10.2001; (1), 25.4.2002. Wadi Shihab, (2), 13.5.2003.

Remarks: This species have a wide range of distribution worldwide (Uygun, 1981). It feeds on scale insects. It was introduced to Syria in 1995 for controlling some pest on citrus trees such Planococcus citri in the 
Syrian Coast. Abboud et al. (2020) reported that this species predates on Protopulvinaria pyriformis.

Host species: Inula viscosa and Rubus idaeus.

Lindorus lophanthae (Blaisdéll, 1892) (Figs. 2D and 3D) Material examined (80): Damascus Rural: Sasaa, (1), 13.8.2001. Dar`a: Wadi Jeelin, 5: (2), 9.5.2002; (2), 29.5.2002; (1), 3.7.2002. Serghaya, (1), 3.10.2002. As`Sweida: Ein alarab, (5), 27.9.2001; (18), 18.10.2001; (13), 29.11.2001; (1), 3.4.2002; (2), 17.7.2002; (1), 1.8.2002; (20), 21.8.2002; (11), 10.9.2002; (2), 23.10.2002.

Remarks: This species have a wide range of distribution almost worldwide (Raimundo and van Harten 2000), with records from Turkey, Jordan and Palestine (Uygun, 1981; Allawi, 1989; Halperin et al., 1995). This species represents a new record to Syria.

Host species: Citrus sp., Cupressus sp. and Nerium oleander.

Tribe Diomini R. D. Gordon, 1999

Diomus rubidus (Motschulsky, 1837) (Figs. 2E and 3E)

Material examined (1): Damascus Rural: Attall, (1), 27.12.2001.

Remarks: Fürsch (1979) recognized three subspecies for this form. Listed by El Hariri (1971) as Scymnus rubidus in Syria. Jalilvand et al. (2014) found that this species predates on Planococcus vovae in Iran.

Host species: Rubus idaeus.

Tribe Hyperaspidini Mulsant, 1846

Hyperaspis (Hyperaspis) femorata (Motschulsky, 1837) (Figs. 2F and 3F)

Material examined (2): As`Sweida: Taamri Mountain, (2), 21.6.2002.

Remarks: This species is distributed in the Caucasus, Armenia, Ukrania (Iablokoff-Khnzorian, 1971) and Bulgaria (Canepari et al., 1985). This species is recorded for the first time in Syria. Aslan and Uygun (2005) reported this species from Turkey to feed on aphids.

Host species: Achillea sp.

Hyperaspis (Hyperaspis) histeroides (Faldermann, 1837) (Figs. 2G and 3G)

Material examined (12): Damascus Rural: Rankus, (1), 11.9.2002. As`Sweida: Qanawat, (1), 19.6.2002. Ghoujygoujyat Mountain, (1), 1.8.2002; (1), 29.4.2003, Orman Mountain, (2), 4.7.2002; (1), 4.8.2003. Taamri Mountain, (2), 28.6.2003; (1), 4.7.2003. Al-Quneitra: Beer Ajam, (1), 27.6.2002. Masehara, (1), 13.8.2001.

Remarks: This species is distributed in the Caucasus, Kazakhstan and Armenia (Iablokoff-Khnzorian, 1971). Reported previously from As-Sweida preying on the almond curl leaf aphid, Brachycaudus amygdalinus (Almatni and Khalil, 2008).

Host species: Achillea sp., Amygdalus sp., Cucurbita pepo, Ficus carica, and Quercus calliprinos.

Hyperaspis (Hyperaspis) syriaca Weise, 1885 (Figs. 2H and $3 \mathrm{H}$ )

Material examined (16): Dar`a: Wadi Jeelin, (4), 9.5.2002; (1), 29.5.2002; (2), 3.7.2002; (9), 22.8.2002.

Remarks: This species is known from Palestine, Syria and Lebanon (Iablokoff-Khnzorian, 1971).

Host species: Vitex agnus-castus.
Tribe Scymnini Mulsant, 1846

Clitostethus arcuatus (Rossi, 1794) (Figs. 2I and 3I).

Material examined (40): Damascus Rural: Attall, (1), 25.10.2001; (20), 20.11.2002. Ma`adamiyeh Al-sham, (4), 6.3.2002; (1), 4.10.2002; (3), 1.11.2002; (1), 4.12.2002; (1), 5.2.2003; (4), 6.3.2003; (2), 9.4.2003; (1), 23.6.2003; (1), 11.7.2003. Damascus: Damascus, (1), 20.10.2002.

Remarks: This species is known from Europe, the Caucasus and Turkey (Uygun 1981). Reported from the Coastal regions of Syria on whiteflies (Al Jundi and Ahmad, 1999) and Palestine (Halperin et al., 1995).

Host species: Citrus sp., Morus alba, Phaseolus vulgaris and Punica granatum.

Nephus (Bipunctatus) bipunctatus (Kugelann, 1794) (Figs. 2J and 3J)

Material examined (97): Damascus Rural: Attall, (2), 26.9.2001; (2), 25.10.2001; (3), 5.9.2002. Wadi AlQaren, (1), 5.9.2002; (1), 10.7.2002. Yabrud, (1), 11.9.2002. Sasaa, (6), 3.10.2001; (2), 12.9.2002; (3), 26.8.2003. Kulaiaa, (1), 7.8.2002, Hadar, (1), 12.9.2002; (1), 26.8.2003. Beet-jen Mazraa Mazraa, (1), 3.10.2001; (1), 26.8.2003. Erneh, (1), 11.7.2002. Beqaasem, (1), 29.8.2002, Rankus Mountain, (4), 11.9.2002, Sarkha, (2), 26.9.2001; (3), 11.9.2002. Al-Qutayfeh, (4), 8.8.2002. Qatana, (1), 14.8.2003. Kharabo, (2), 2.4.2003; (2), 6.8.2003. Serghaya, (1), 5.9.2002. Ma`adamiyeh Al-sham, (2), 30.8.2002; (3), 9.9.2002; (2), 4.10.2002; (2), 11.7.2003; (2), 13.8.2003. As`Sweida: Al-Kafr, (1), 18.10.2001; (1), 23.10.2002. Ein alarab, (1), 17.7.2002. Orman, (1), 27.9.2001. Orman Mountain, (1), 9.4.2003. Taamri Mountain, (1), 8.8.2003. Qanawat, (1), 11.9.2001; (1), 20.9.2001; (2), 13.3.2002; (1), 21.8.2002. Shahba, (1), 13.3.2002. Salkhad, (1), 22.11.2001. Taima, (2), 11.9.2001. Alqrayya, (2), 10.9.2001, Dar`a: Wadi Jeelin, (1), 7.2.2002; (1), 22.8.2002. Zeizon, (1), 22.8.2002; (1), 13.11.2002. Sheikh Miskin, (2), 22.7.2003. Sahm aljoulan, (10), 4.9.2001. Al-Quneitra: Beer Ajam, (2), 12.9.2002; (2), 2.10.2002; (1), 19.3.2003. Trounja, (1), 29.8.2002.

Remarks: This is a Palaearctic species with a wide range of distribution in Central Asia, North Africa reaching Japan (Gourreau, 1974; Kuznetsov, 1997). Reported previously from As-Sweida preying on the almond curl leaf aphid, Brachycaudus amygdalinus (Almatni and Khalil, 2008).

Host species: Amygdalus sp., Prunus armeniaca, Cedrus libani, Citrus sp., Ficus carica, Hibiscus esculentus, Malus communis, Morus alba, Phaseolus vulgaris, Pinus sp., Populus sp., Prunus sp., Prunus avium, Prunus vulgaris, Punica granatum, Pyrus communis, Quercus calliprinos, Urtica sp., Vitex agnus-castus, Vitis vinifera, and thistle plants.

Nephus (Bipunctatus) includens (Kirsch, 1870) (Figs. 2K and 3K)

Material examined (2): Dar`a: Wadi Jeelin, (1), 7.2.2002. Sahm al-joulan, (1), 4.9.2001.

Remarks: This species is distributed in some the Mediterranean area and Saudi Arabia (Uygun, 1981). Listed in Syria by El Hariri (1968) and recorded from the Coastal area on Planococcus citri (Aslan, 2001). It has been recorded as a predator of the mealybug Nipaecoccus viridis on different hosts in Iran (Fallahzadeh et al., 2013). 
Other species of this genus in Iran were found to prey on Planococcus vovae (Jalilvand et al., 2014).

Nephus (Nephus) ludyi (Weise, 1879) (Figs. 2L and 3L)

Material examined (115): Damascus Rural: Erneh, (1), 29.8.2002. Beqaasem, (1), 29.8.2002. Wadi Al-Qaren, (9), 17.11.2001; (3), 19.9.2001; (2), 5.9.2002; (3), 14.3.2002. Al-Quneitra: Jubata Al-Khashab, (3), 8.11.2001; (1), 20.12.2001; (3), 20.3.2002; (4), 28.2.2002; (2), 10.4.2002; (2), 25.4.2002. Beer Ajam, (6), 20.3.2002. As`Sweida: Al-Kafr, (11), 20.9.2001; (10), 18.10.2001; (31), 29.11.2001; (1), 21.3.2002; (3), 24.4.2002; (1), 17.7.2002; (1), 1.8.2002; (1), 29.11.2002; (1), 12.12.2002. Ein alarab, (1), 17.7.2002; (1), 1.8.2002; (1), 21.8.2002. Ghoujygoujyat Mountain, (1), 22.10.2002; (2), 4.8.2003. Orman Mountain, (3), 4.8.2003. Taamri Mountain, (1), 5.8.2003. Qanawat, (2), 20.9.2001; (1), 13.3.2002; (1), 3.4.2002; (1), 11.4.2002.

Remarks: This species is known from southern France and the Mediterranean region (Gourreau, 1974). Recorded from Jordan (Allawi, 1989). This species is recorded for the first time in Syria.

Host species: Amygdalus sp., Prunus armeniaca, Juglans regia, Malus communis, Prunus avium, Prunus vulgaris, Pyrus communis and Quercus calliprinos.

Nephus (Nephus) merkli Fürsch, 1994 (Figs. 2M and 3M)

Material examined (115): Damascus Rural: Seydnaya, (3), 21.5.2003. Qatana, (2), 14.8.2003. Attall, (7), 26.9.2001; (4), 25.10.2001; (1), 21.11.2001; (1), 10.7.2002. Kharabo, (46), 22.8.2001; (4), 26.9.2001; (10), 25.10.2001; (3), 21.11.2001; (2), 27.3.2002; (2), 11.7.2002; (1), 24.7.2002; (9), 8.8.2002; (2), 11.9.2002; (2), 25.9.2002; (4), 9.10.2002. Ma`adamiyeh Al-sham, (1), 4.10.2002; (1), 1.11.2002; (1), 23.6.2003; (1), 6.7.2003; (2), 23.7.2003; (1), 9.8.2003; (1), 13.8.2003. Dar`a: Wadi Jeelin, (1), 3.7.2002; (1), 18.7.2002. Zeizon, (1), 23.4.2003. Al-Quneitra: Masehara, (1), 13.8.2001.

Remarks: Described originally from Palestine (Fürsch, 1994), and very similar to Nephus quadrimaculatus pictus. This species is recorded for the first time in Syria.

Host species: Citrus sp., Cupressus sp., Ficus carica, Juglans regia, Ligustrum sp., Morus alba, Nerium oleander, Olea europaea, Prunus avium, Punica granatum, Solanum melongena, Urtica sp. and Zea mays.

Nephus (Nephus) quadrimaculatus Herbst, 1783 (Figs. $2 \mathrm{~N}$ and $3 \mathrm{~N}$ )

Material examined (265): Damascus Rural: Beet-jen Mazraa, (3), 10.4.2002. Durbol, (1), 29.8.2002. Beqaasem, (3), 21.9.2001; (4), 209.8.2002. Wadi Al-Qaren, 12: (2), 17.11.2001; (8), 19.8.2001; (2), 19.9.2001. Sasaa, (1), 13.8.2001; (2), 2.5.2002; (1), 12.9.2002. Kulaiaa, (5), 7.8.2002. Addimass, (1), 30.4.2003. Qatana, (1), 14.8.2003. Attall, (13), 3.9.2001; (12), 26.9.2001; (10),
25.10.2001; (2), 21.11.2001; (1), 27.12.2001; (1), 14.3.2002; (1), 26.6.2002; (11), 10.7.2002; (25), 5.9.2002; (1), 24.8.2003. Kharabo, (1), 25.10.2001; (1), 21.11.2001; (1), 27.3.2002; (1), 25.9.2002; (11), 2.7.2003. Ma`adamiyeh Al-sham, (1), 6.12.2001; (1), 6.3.2002; (1), 30.8.2002; (1), 4.10.2002; (1), 1.11.2002; (1), 11.7.2003; (3), 23.7.2003; (5), 27.7.2003; (1), 9.8.2003. Al-Quneitra: Masehara, (1), 13.8.2001. Jubata Al-Khashab, (1), 20.3.2002; (4), 28.2.2002; (5), 16.4.2003; (1), 26.8.2003. Hadar, (5), 10.9.2001. Ophania, (1), 10.9.2001. Beer Ajam, (8), 20.3.2002; (5), 12.9.2002; (3), 2.10.2002; (2), 19.3.2003. As`Sweida: Al-Kafr, (2), 18.10.2001; (1), 9.5.2002; (1), 17.7.2002. Ein alarab, (1), 6.6.2002; (1), 23.10.2002. Orman Mountain, (12), 27.9.2001; (1), 21.8.2002. Orman (1), 4.7.2002. Taamri Mountain, (1), 5.8.2003. Qanawat, (1), 12.8.2001; (3), 20.9.2001; (1), 13.3.2002. Shahba, (4), 23.3.2002. Salkhad, (7), 22.11.2001. Baka, (1), 20.9.2001. Taima, (4), 11.9.2001. Alqrayya, (12), 20.9.2001. Dar`a: Wadi Jeelin, (3), 21.9.2001; (2), 24.10.2001; (4), 22.11.2001; (2), 7.2.2002; (1), 29.5.2002; (5), 3.7.2002; (2), 18.7.2002; (9), 22.8.2002. Zeizon, (1), 20.12.2001; (1), 25.4.2002; (3), 2.10.2002.

Remarks: This is a Palaearctic species (Gourreau, 1974). Listed by El Hariri (1971) as Scymnus quadrimaculatus pictus in Syria. Reported from Palestine, the Golan Heights, Mount Hermon and Lebanon (Canepari and Tedeschi, 1977; Halperin et al., 1995). It feeds on scale insects (Klausnitzer and Klausnitzer, 1986).

Host species: Amygdalus sp., Citrus sp., Cupressus sp., Ficus carica, Juglans regia, Morus alba, Nerium oleander, Olea europaea, Prunus vulgaris, Pinus sp., Pistacia vera, Phaseolus vulgaris, Populus sp., Prunus armeniaca, Prunus avium, Punica granatum, Pyrus communis, Quercus calliprinos, Rubus idaeus, Schinus molle, and thistle plants.

Nephus (Sidis) caucasicus Weise, 1929 (Figs. 20 and 30)

Material examined (1): Damascus Rural: Attall, (1), 27.12.2001.

Remarks: This species is recorded for the first time in Syria. Previously recorded from Turkey and the Cuacases (Fürsch, 1965).

Host species: Urtica sp.

Nephus (Sidis) hiekei Fürsch, 1965 (Figs. 2P and 3P)

Material examined (2): Damascus Rural: Jeesrin, (1), 29.12.2002. Dar`a: Zeizon, (1), 20.12.2001.

Remarks: This species is recorded for the first time in Syria. This species is widely distributed throughout southern Europe and the Middle East (Raimundo and van Harten, 2000), with records from Lebanon and Palestine on Pseudococcus citri (Fürsch, 1967b).

Host species: Urtica sp. 

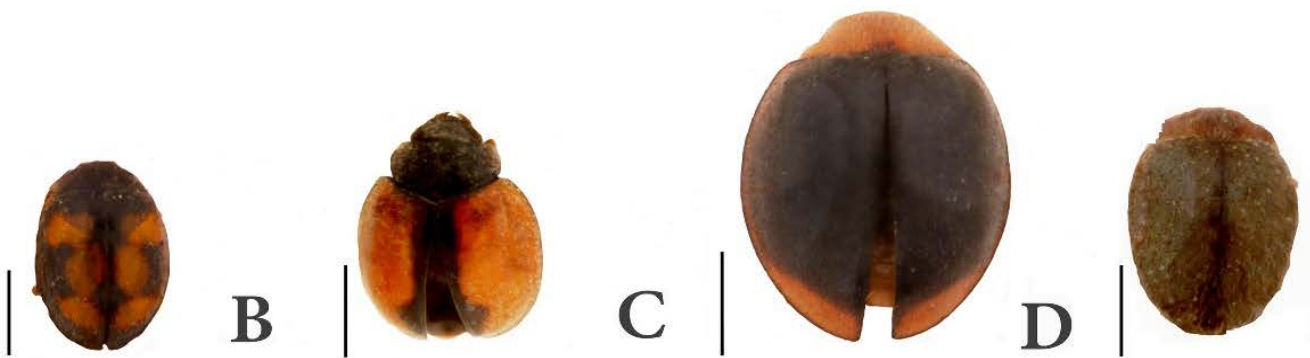

$\mathbf{E}$
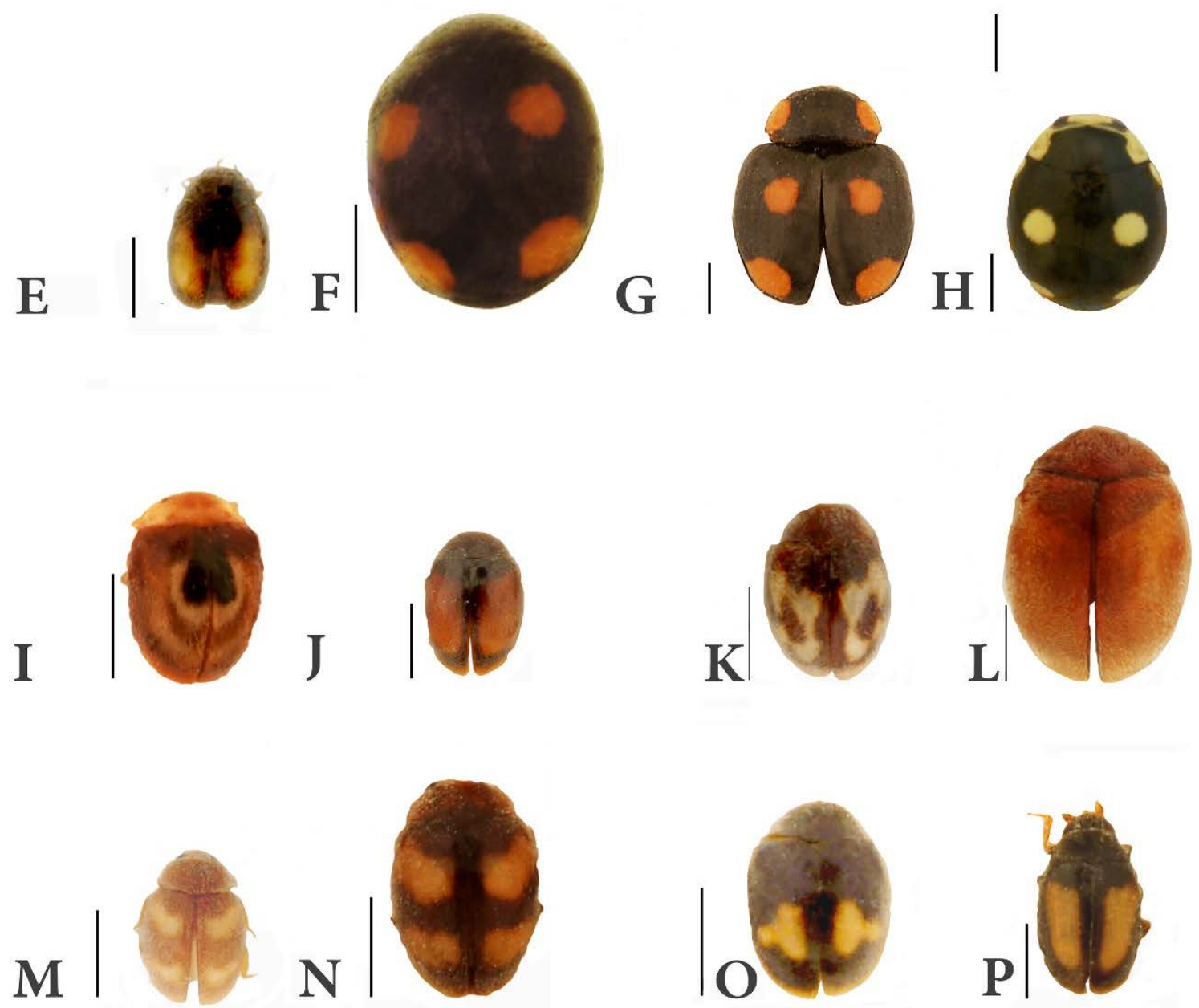

Figure 2: A. Pharoscymnus ovoideus. B. Pharoscymnus setulosus. C. Cryptolaemus montrouzieri. D. Lindorus lophantae. E. Diomus rubidus. F. Hyperaspis (Hyperaspis) femorata. G. Hyperaspis (Hyperaspis) histeroides. H. Hyperaspis (Hyperaspis) syriaca. I. Clitostethus arcuatus. J. Nephus (Bipunctatus) bipunctatus. K. Nephus (Bipunctatus) includens. L. Nephus (Nephus) ludyi. M. Nephus (Nephus) merkli. N. Nephus (Nephus) quadrimaculatus. O. Nephus (Sidis) caucasicus. P. Nephus (Sidis) hiekei. Scale bar=1 mm. 
A
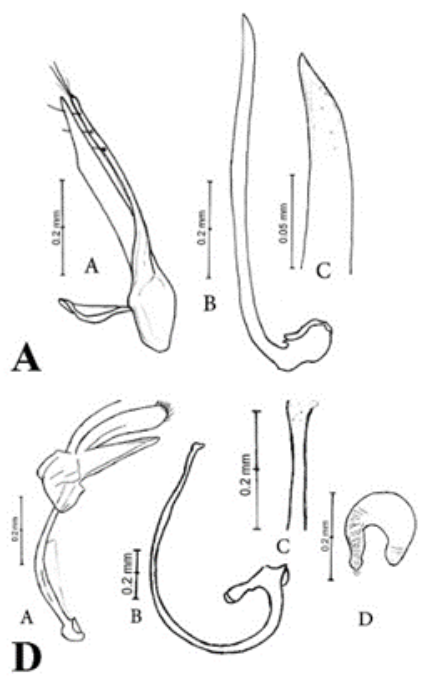

D

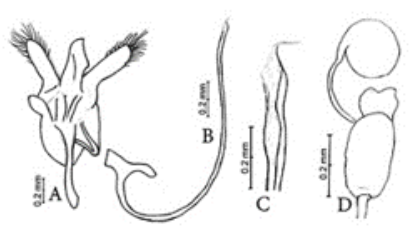

G
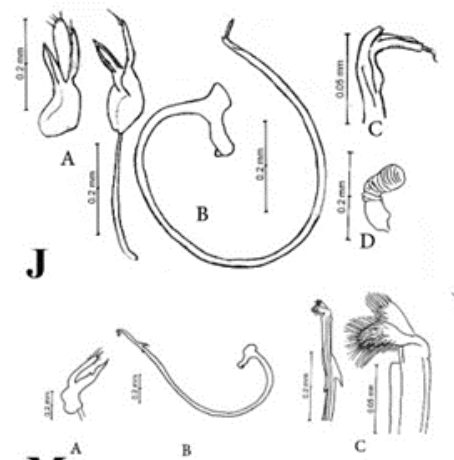

B

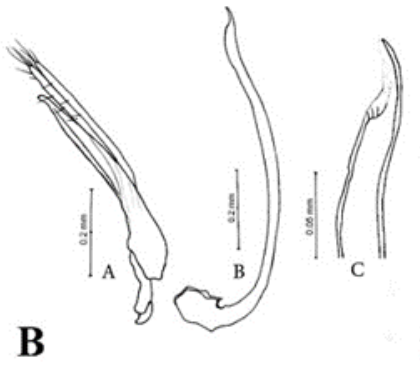

C
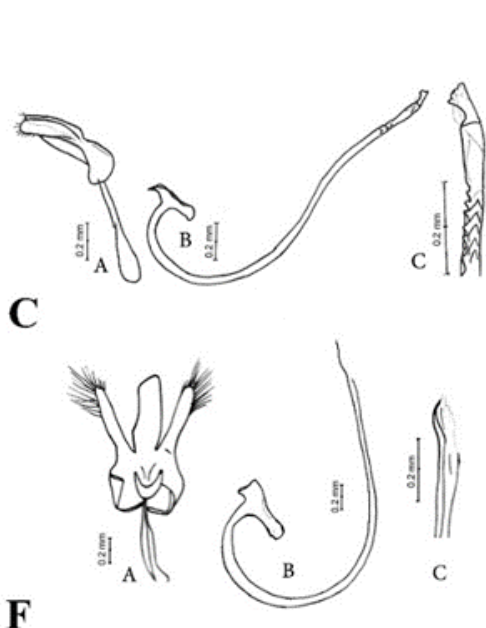

E

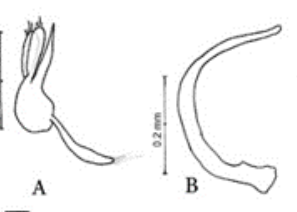

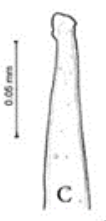

F

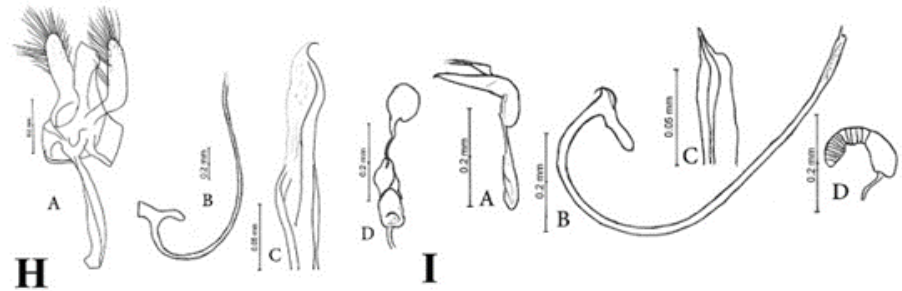

H

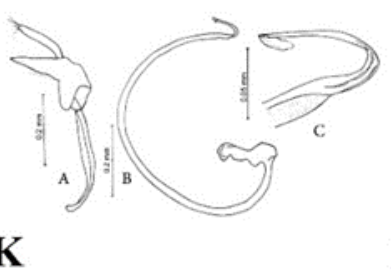

K

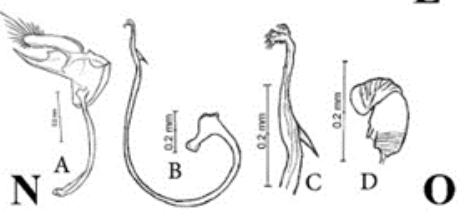

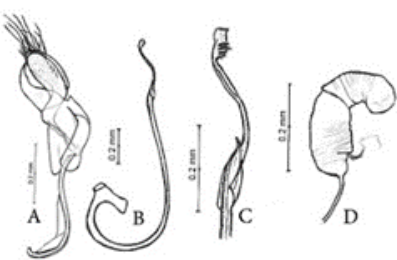

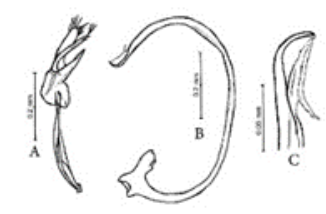

$\mathbf{M}^{\wedge}$
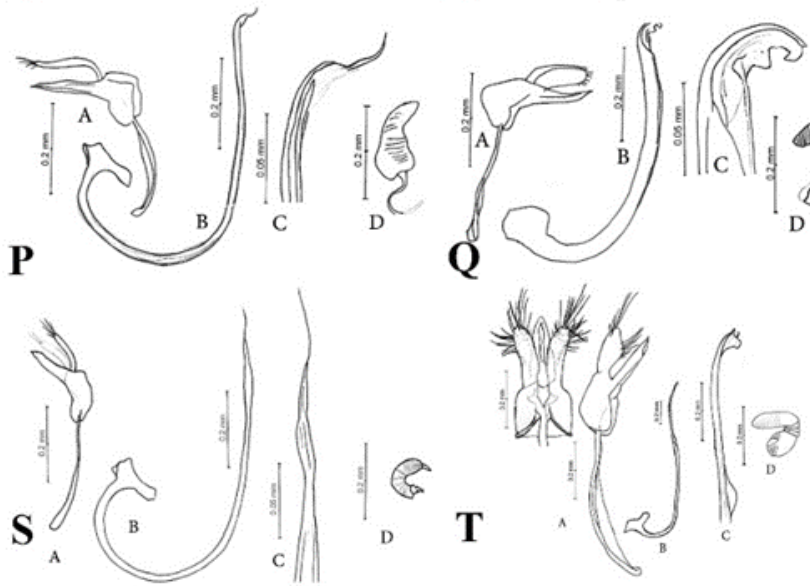

R
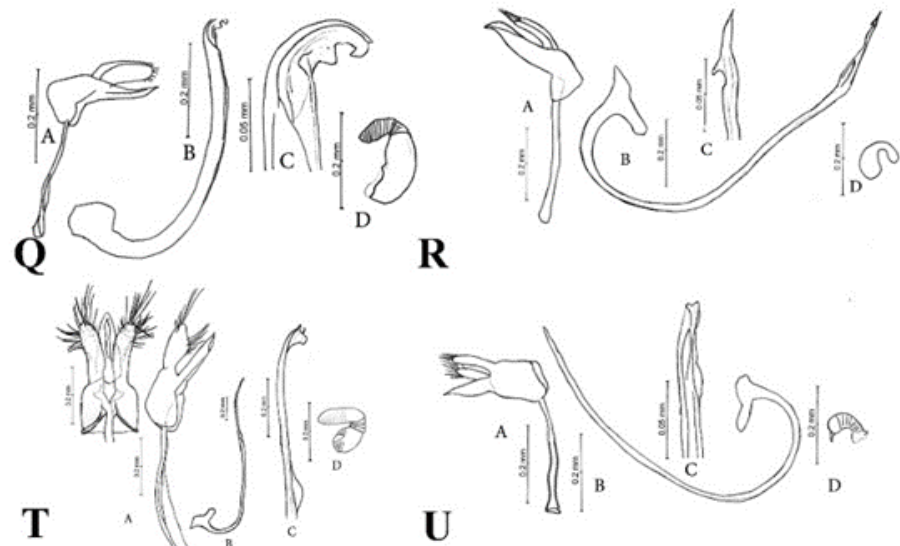

Figure 3: A. Pharoscymnus ovoideus. B. Pharoscymnus setulosus. C. Cryptolaemus montrouzieri. D. Lindorus lophantae. E. Diomus rubidus. F. Hyperaspis (Hyperaspis) femorata. G. Hyperaspis (Hyperaspis) histeroides. H. Hyperaspis (Hyperaspis) syriaca. I. Clitostethus arcuatus. J. Nephus (Bipunctatus) bipunctatus. K. Nephus (Bipunctatus) includens. L. Nephus (Nephus) ludyi. M. Nephus (Nephus) merkli. N. Nephus (Nephus) quadrimaculatus. O. Nephus (Sidis) caucasicus. P. Nephus (Sidis) hiekei. Q. Nephus (Sidis) kreissli. R. Scymnus (Mimopullus) pharaonis. S. Scymnus (Mimopullus) flagellisiphonatus. T. Scymnus (Mimopullus) flavicollis. U. Scymnus (Mimopullus) marinus. Key to darwings: $\mathrm{A}=$ Tegmen, $\mathrm{B}=$ Penis, $\mathrm{C}=$ Penis apex, $\mathrm{D}=$ Spermatheca. 
Nephus (Sidis) kreissli Fürsch and Uygun, 1980 (Figs. 4A and 3Q)

Material examined (27): Damascus Rural: Attall, (2), 26.9.2001; (1), 25.10.2001; (1), 21.11.2001; (4), 14.3.2002; (9), 8.5.2002; (2), 26.6.2002; (1), 5.9.2002. Ma`adamiyeh Al-sham, (1), 23.6.2003. Dar`a: Wadi Jeelin, (1), 22.11.2001; (1), 18.7.2002; (1), 22.8.2002. Zeizon, (1), 22.8.2002; (2), 23.4.2003.

Remarks: This species is recorded for the first time in Syria. This species was known from Turkey (Fürsch and Uygun, 1980).

Host species: Citrus sp., Ficus carica, Inula viscosa, Malus communis, Morus alba, Nerium oleander, Punica granatum, Populus sp., Rubus idaeus, Urtica sp. and Vitex agnus-castus.

Scymnus (Mimopullus) pharaonis Motschulsky, 1851 (Figs. 4B and 3R)

Material examined (317): Damascus Rural: Wadi Al-Qaren, (6), 19.9.2001; (1), 5.9.2002; (8), 10.7.2002; (1), 15.6.2002; (1), 17.11.2002. Yabrud, (1), 8.8.2002. Kharabo, (1), 26.9.2001; (2), 25.10.2001. Ma`adamiyeh Al-sham, (3), 6.12.2001; (4), 28.6.2002; (11), 30.8.2002; (8), 9.9.2002; (10), 4.10.2002; (2), 1.11.2002; (1), 23.6.2003; (10), 11.7.2003; (2), 23.7.2003; (1), 13.8.2003. Sasaa, (1), 3.10.2002; (3), 12.9.2002. Kulaiaa, (17), 7.8.2002. Dorin, (1), 10.7.2002. Beet-jen Mazraa, (5), 3.10.2001; (1), 8.11.2001; (1), 7.8.2002. Erneh, (7), 21.9.2001. Beqaasem, (2), 21.9.2001; (1), 11.7.2002; (3), 29.8.2002. Mugr Almeer, (1), 26.8.2003. Kafr- Hawar, (1), 7.8.2002. Deir Al-ashairr, (1), 17.9.2002. Addimass, (1), 17.9.2002. Al-Qutayfeh, (3), 8.8.2002. Qatana, (2), 14.8.2003. Assaida Zainab, (1), 13.3.2002. Ashiphonieh, (1), 25.9.2002. Attall, (1), 26.9.2001; (2), 26.6.2002; (3), 10.7.2002; (1), 20.11.2002. As`Sweida: Al-Kafr, (3), 20.9.2001; (2), 29.11.2001. Ein alarab, (1), 7.8.2001; (2), 17.7.2002; (1), 1.8.2002. Orman, (1), 27.9.2001; (3), 4.7.2002, (3), 17.7.2002; (1), 6.6.2003. Ghoujygoujyat Mountain, (1), 4.7.2002; (4), 1.8.2002; (2), 21.8.2002; (1), 10.9.2002; (4), 23.10.2002; (3), 4.8.2003. Taamri Mountain, (1), 17.7.2002; (2), 4.7.2003; (1), 14.7.2003; (4), 25.7.2003; (7), 5.8.2003; (4), 8.8.2003. Orman Mountain, (4), 4.8.2003. Qanawat, (2), 11.9.2001; (12), 20.9.2001; (1), 19.6.2002; (3), 1.8.2002; (3), 21.8.2002. Shahba, (1), 19.6.2002. Alqrayya, (14), 20.9.2001. Dar`a: Jeelin, (9), 3.7.2002, Wadi Jeelin, (4), 18.7.2002; (1), 22.8.2002. Zeizon, (1), 29.5.2002; (1), 22.8.2002; (2), 2.10.2002; (2), 13.11.2002; (1), 19.3.2003. Mzireeb, (1), 9.5.2002. Nahaj, (9), 4.10.2001; (5), 24.10.2001. Tafas, (1), 4.9.2001. Sahm al-joulan, (12), 4.9.2001. Namer, (3), 20.6.2002. Al-Quneitra: Beer Ajam, (2), 20.3.2002; (1), 27.6.2002; (9), 12.9.2002; (10), 2.10.2002. Jubata AlKhashab, (1), 10.9.2001; (1), 20.3.2002; (1), 10.4.2002. Hadar, (2), 31.7.2002; (9), 12.9.2002; (1), 26.8.2003.

Remarks: Its distribution range extends from Central Asia to North Africa to Europe (Gourreau, 1974; Uygun, 1981). Scymnus (Mimopullus) araraticus IablokoffKhnzorian, 1969 is now considered a synonym for $S$. pharaonis (Kovár 2007). It was recorded by Almatni and Khalil (2008).

Host species: Aloysia citrodora, Amygdalus sp., Prunus armeniaca, Cedrus libani, Citrus sp., Cupressus sp., Faba vulgaris, Ficus carica, Hibiscus esculentus,
Juglans regia, Lonicera japonica, Malus communis, Medicago sativa, Morus alba, Olea europaea, Pinus sp., Phaseolus vulgaris, Pistacia vera, Platanus orientalis, Populus sp., Prunus avium, Prunus mahaleb, Prunus persica, Punica granatum, Pyrus communis, Quercus calliprinos, Triticum sp. and thistle plants.

Scymnus (Mimopullus) flagellisiphonatus Fürsch, 1969 (Figs. 4C and 3S)

Material examined (26): Al-Quneitra: Beer Ajam, (2), 20.3.2002. Jubata Al-Khashab, (1), 25.4.2002; (1), 16.5.2002. Trounja, (1), 10.4.2002. Masehara, (1), 2.5.2002. Damascus Rural: Attall, (1), 26.9.2001. Beetjen Mazraa, (1), 10.4.2002. Kharabo, (1), 23.5.2002. Serghaya, (1), 30.5.2002, Ma`adamiyeh Al-sham, (1), 12.4.2002; (1), 13.8.2003. As`Sweida: Al-Kafr, (1), 9.5.2002, Orman, (1), 4.7.2002. Ghoujygoujyat Mountain, (1), 4.7.2002. Qanawat, (5), 11.4.2002. Dar`a: Jeelin, (1), 10.8.2001; (1), 3.7.2002. Wadi Jeelin, (1), 28.3.2002. Zeizon, (1), 10.4.2002, Sabsaba, (2), 10.4.2002.

Remarks: Known from the Mediterranean Region (Uygun, 1981) and recorded from Wadi Al Qaren in Syria by Kabakibi (1993). It feeds on aphids (Canepari, 1991).

Host species: Amygdalus sp., Juglans regia, Medicago sativa, Pinus sp., Pistacia vera, Prunus avium, Punica granatum and Quercus calliprinos.

Scymnus (Mimopullus) flavicollis Redtenbacher, 1844 (Figs. 4D and 3T)

Material examined (416): Damascus Rural: Kharabo, (3), 22.8.2001; (1), 26.9.2001; (1), 25.10.2001; (1), 15.5.2002; (23), 23.5.2002; (6), 5.6.2002; (8), 11.7.2002; (2), 24.7.2002; (5), 11.9.2002; (3), 25.9.2002; (6), 9.10.2002; (13), 25.5.2003; (10), 2.7.2003; (2), 6.8.2003. Serghaya, (6), 7.11.2001; (4), 10.7.2002; (14), 24.7.2002; (18), 14.8.2002; (20), 5.9.2002; (21), 17.9.2002; (12), 3.10.2002; (3), 7.11.2002; (1), 10.4.2003; (1), 22.5.2003; (7), 26.6.2003; (12), 29.7.2003. Ma`adamiyeh Al-sham, (1), 23.5.2002; (10), 28.6.2002; (7), 30.8.2002; (2), 9.9.2002; (4), 4.10.2002; (2), 1.11.2002; (3), 19.5.2003; (13), 23.6.2003; (19), 11.7.2003; (8), 23.7.2003; (1), 9.8.2003; (3), 13.8.2003. Yabrud, (2), 25.10.2001. Sasaa, (1), 31.7.2002; (1), 12.9.2002. Dorin, (2), 10.7.2002. Beet-jen Mazraa, (2), 7.8.2002; (6), 29.8.2002. Zarzar, (3), 19.9.2001. Al Hameh, (9), 14.6.2003. Azzabadani, (2), 27.6.2002. Sarkha, (1), 26.6.2002. Qatana, (3), 5.7.2002. Airport Street, (1), 28.8.2001. Haran Al-awameed, (3), 24.7.2002. Assaida Zainab, (6), 11.9.2001; (2), 18.10.2001. Ashiphonieh, (2), 25.9.2002. Attall, (5), 26.9.2001; (1), 25.10.2001; (8), 26.6.2002; (26), 10.7.2002; (1), 5.9.2002. As`Sweida: Ein alarab, (3), 28.6.2002; (3), 1.8.2002; (1), 23.10.2002; (5), 4.8.2003. Orman, (1), 27.9.2001; (18), 4.7.2002; (1), 11.7.2002; (5), 17.7.2002; (2), 12.6.2003. Orman Mountain, (1), 4.7.2002. Dar`a: Wadi Jeelin, (1), 22.11.2001; (1), 22.8.2002. Jeelin, (2), 3.7.2002. Namer, (3), 20.6.2002. Al-Quneitra: Beer Ajam, (3), 12.9.2002; (1), 2.10.2002. Hadar, (1), 10.9.2002. Trounja, (1), 28.2.2002.

Remarks: Recorded from Iran, Palestine and the Golan Heights (Ahmadi and Yazdani 1993; Halperin et al., 1995). Listed for Syria by El Hariri (1968 and 1971).

Host species: Althaea officinalis, Amygdalus sp., Prunus armeniaca, Capsicum annum, Cedrus libani, 
Citrus sp., Cucumis melo, Cucurbita pepo, Cydonia vulgaris, Dacus carota, Elaeagnus angustifolia, Euphorpia sp., Ficus carica, Foeniculum vulgare, Glycyrrhiza glabra,, Hypericum trinquertifolium, Inula viscosa, Juglans regia, Malus communis, Medicago sativa, Morus alba, Nerium oleander, Oncium sp., Phaseolus vulgaris, Populus sp., Prunus avium, Prunus mahaleb, Punica granatum, Pyrus communis, Quercus calliprinos, Rosa sp., Rubus idaeus, Solanum melongena, Schinus molle, Triticum sp., Urtica sp., Zea mays, and thistle plants.

Scymnus (Mimopullus) marinus Mulsant, 1850 (Figs. 4E and $3 \mathrm{U}$ )

Material examined (109): Damascus Rural: Ma`adamiyeh Al-sham, (2), 6.12.2001; (3), 28.6.2002; (5), 30.8.2002; (4), 9.9.2002; (8), 4.10.2002; (13), 1.11.2002; (5), 4.12.2002; (1), 5.2.2003; (6), 11.7.2003; (2), 23.7.2003; (2), 13.8.2003. Assaida Zainab, (1), 13.3.2002. As`Sweida: Taamri Mountain, (4), 6.6.2003; (6), 20.6.2003; (5), 4.7.2003; (1), 14.7.2003; (4), 25.7.2003; (9), 5.8.2003; (6), 8.8.2003. Orman, (3), 6.6.2003. Ghoujygoujyat Mountain, (2), 12.6.2003. Shahba, (1), 19.6.2002; (3), 11.12.2002. Barad, (1), 18.10.2001. Baka, (1), 20.9.2001. Taima, (7), 11.9.2001. Tal-loz, (3), 12.8.2001. Dar`a: Wadi Jeelin, (1), 22.11.2001.

Remarks: This species is recorded for the first time in Syria. It is distributed in Europe and North Africa (Gourreau, 1974) and was recorded from Palestine (Halperin et al., 1995). Scymnus mediterraneus IablokoffKhnzorian, 1972 is considered as a synonym (Kovář, 2007).

Host species: Aloysia citrodora, Amygdalus sp., Biota orientalis, Citrus sp., Cupressus sp., Faba vulgaris, Juglans regia, Olea europaea, Pinus sp., Pistacia vera, Phaseolus vulgaris, Prunus armeniaca, P. avium and Punica granatum.

Scymnus (Parapullus) abietis (Paykull, 1798) (Figs. 4F and 5A)

Material examined (2): Damascus Rural: Wadi AlQaren, (1), 19.8.2001. As`Sweida: Orman, (1), 17.7.2002.

Remarks: This species is recorded for the first time in Syria. Distribution of this species extends from Europe, North Africa to Mongolia and the Russian Far East (Gourreau, 1974, Kuznetsov, 1997; Kováŕ, 2007).

Host species: Pinus sp. and Quercus calliprinos.

Scymnus (Pullus) apetzi Mulsant, 1846 (Figs. 4G and 5B)

Material examined (403): Damascus Rural: Kharabo, (1), 22.8.2001; (1), 26.9.2001; (1), 25.10.2001; (11), 23.5.2002; (1), 5.6.2002; (2), 11.7.2002; (5), 24.7.2002; (1), 11.9.2002; (7), 25.9.2002; (4), 9.10.2002; (9), 25.5.2003; (8), 2.7.2003; (3), 6.8.2003. Serghaya, (2), 7.11.2001; (2), 30.5.2002; (1), 27.6.2002; (4), 10.7.2002; (6), 24.7.2002; (22), 14.8.2002; (17), 15.9.2002; (32), 17.9.2002; (18), 3.10.2002; (1), 7.11.2002; (1), 10.4.2003; (1), 22.5.2003; (6), 26.6.2003; (13), 29.7.2003. Ma`adamiyeh Al-sham, (12), 28.6.2002; (3), 30.8.2002; (1), 9.9.2002; (1), 4.10.2002; (1), 19.5.2003; (7), 23.6.2003; (11), 11.7.2003; (3), 23.7.2003; (1), 9.8.2003. Wadi Al-Qaren, (2), 10.7.2002. Sasaa, (1), 2.5.2002; (1), 12.6.2002; (2), 31.7.2002. Kulaiaa, (1), 7.8.2002. Dorin, (1), 24.8.2003. Beet-jen Mazraa, (2), 7.8.2002; (6), 29.8.2002. Erneh, (2), 11.7.2002. Beqaasem, (1),
21.9.2001; (1), 11.7.2002. Mugr Almeer, (1), 26.8.2003. Zarzar, (2), 19.9.2001. Deir Al-ashairr, (1), 17.10.2001. Al-Hameh, (7), 16.4.2003. Azzabadani, (5), 27.6.2002; (1), 14.6.2003. Al-Qutayfeh, (3), 8.8.2002. Qatana, (2), 14.8.2003. Ashiphonieh, (1), 25.9.2002. Attall, (3), 26.9.2001; (2), 25.10.2001; (3), 8.5.2002; (3), 26.6.2002; (20), 10.7.2002; (2), 5.9.2002; (7), 14.8.2003. As`Sweida: Ein alarab, (1), 27.9.2001; (7), 28.6.2002; (2), 4.7.2002; (15), 1.8.2002; (4), 23.10.2002; (3), 22.7.2003; (8), 4.8.2003. Orman Mountain, (1), 1.8.2001; (7), 1.8.2002. Orman, (1), 27.9.2001; (1), 24.6.2002; (21), 4.7.2002; (1), 17.7.2002; (1), 10.7.2002; (1), 12.6.2003. Taamri Mountain, (1), 1.8.2002, Ghoujygoujyat Mountain, (5), 23.10.2002. Qanawat, (1), 18.10.2001; (1), 13.3.2002; (1), 1.8.2002. Alqrayya, (1), 20.9.2001. Dar`a: Jeelin, (2), 3.7.2002. Wadi Jeelin, (2), 3.7.2002. Zeizon, (1), 29.5.2002; (1), 22.8.2002. Sheikh Miskin, (2), 30.8.2001; (1), 27.5.2003. Nahaj, (3), 4.10.2001. Namer, (1), 26.6.2002. Al-Quneitra: Beer Ajam, (2), 12.9.2002. Jubata Al-Khashab, (1), 26.8.2003.

Remarks: This species has a wide range of distribution in Central Europe, the Mediterranean Region, including North Africa and Middle Asia (Gourreau, 1974). It recorded by Almatni and Khalil (2008).

Host species: Althaea officinalis, Amygdalus sp., Prunus armeniaca, Capsicum annum, Cedrus libani, Citrus sp., Cucumis melo, Cucurbita pepo, Cydonia vulgaris, Dacus carota, Elaeagnus angustifolia, Euphorpia sp., Ficus carica, Foeniculum vulgare, Glycyrrhiza glabra,, Hypericum trinquertifolium, Inula viscosa, Juglans regia, Malus communis, Medicago sativa, Morus alba, Nerium oleander, Oncium sp., Phaseolus vulgaris, Populus sp., Prunus avium, Prunus mahaleb, Punica granatum, Pyrus communis, Quercus calliprinos, Rosa sp., Rubus idaeus, Solanum melongena, Schinus molle, Triticum sp., Urtica sp., Zea mays, and thistle plants.

Scymnus (Pullus) auritus Thunberg, 1795 (Figs. 4H and 5C)

Material examined (5): Al-Quneitra: Jubata AlKhashab, (1), 16.5.2002. Damascus Rural: Wadi AlQaren, (2), 5.9.2002. Sasaa, (1), 12.9.2002. Erneh, (1), 11.7.2002.

Remarks: This species is recorded for the first time in Syria. It is distributed in the largest part of temperate Eurasia (Gourreau, 1974; Kovář, 2007). Uygun (1981) reported this species from Turkey It feeds on aphids.

Host species: Amygdalus sp., Malus communis and Quercus calliprinos.

Scymnus (Pullus) fraxini Mulsant, 1850 (Figs. 4I and 5D)

Material examined (62): Al-Quneitra: Beer Ajam, (25), 12.9.2002; (17), 2.10.2002. Jubata Al-Khashab, (4), 10.9.2001; (1), 8.11.2001; (8), 25.4.2002; (5), 16.5.2002; (1), 31.7.2002; (1), 29.8.2002.

Remarks: This species is recorded for the first time in Syria. Reported in the Balkans and other parts of Europe (Gourreau, 1974).

Host species: Quercus calliprinos.

Scymnus (Pullus) subvillosus (Goeze, 1777) (Figs. 4J and 5E)

Material examined (316): Damascus Rural: Wadi Al-Qaren, (1), 19.9.2002; (2), 5.9.2002. Nasseria, (1), 
5.6.2002. Beet-jen Mazraa, (1), 10.4.2002; (1), 7.8.2002. Erneh, (2), 11.7.2002. Beqaasem, (1), 21.9.2001. Deir Alashairr, (4), 17.10.2001; (1), 28.11.2002. Addimass, (1), 30.4.2003. Rankus Mountain (1), 21.5.2003. Attall, (1), 10.7.2002. Kharabo, (4), 22.8.2001; (2), 25.10.2001; (1), 21.11.2001; (2), 23.5.2002; (1), 11.7.2002; (3), 8.8.2002; (1), 9.10.2002; (1), 27.11.2002; (3), 13.5.2003; (3), 25.5.2003; (2), 2.7.2003. Serghaya, (1), 5.9.2002. Ma`adamiyeh Al-sham, (4), 23.5.2002; (1), 4.12.2002; (1), 9.4.2003. Al-Quneitra: Beer Ajam, (6), 20.3.2002; (1), 27.6.2002; (1), 12.9.2002; (2), 19.3.2003. Jubata AlKhashab, (1), 20.3.2002; (1), 10.4.2002; (2), 25.4.2002; (2), 16.5.2002; (1), 16.4.2003. Trounja, (1), 10.4.2002. As`Sweida: Al-Kafr, (11), 18.10.2001; (20), 29.11.2001; (11), 24.4.2002; (6), 9.5.2002; (3), 6.6.2002; (1), 17.7.2002; (4), 23.10.2002; (3), 11.5.2003; (1), 12.6.2003. Ein alarab, (4), 9.5.2002; (2), 28.6.2002; (1), 4.7.2002; (4), 17.7.2002; (4), 1.8.2002; (1), 10.9.2002; (2), 12.6.2002. Orman Mountain, (3), 11.4.2002; (1), 9.4.2003. Orman, (4), 21.6.2002; (3), 24.6.2002; (12), 4.7.2002; (6), 17.7.2002; (8), 6.6.2003; (3), 4.8.2003. Taamri Mountain, (1), 21.6.2002; (1), 17.7.2002; (1), 1.8.2002; (2), 21.8.2002; (3), 6.6.2003; (1), 20.6.2003; (1), 28.6.2003; (6), 4.7.2003; (12), 14.7.2003; (6), 25.7.2003; (1), 5.8.2003; (2), 8.8.2003. Ghoujygoujyat Mountain, (2), 4.7.2002; (9), 23.10.2002; (4), 4.8.2003. Qanawat, (7),
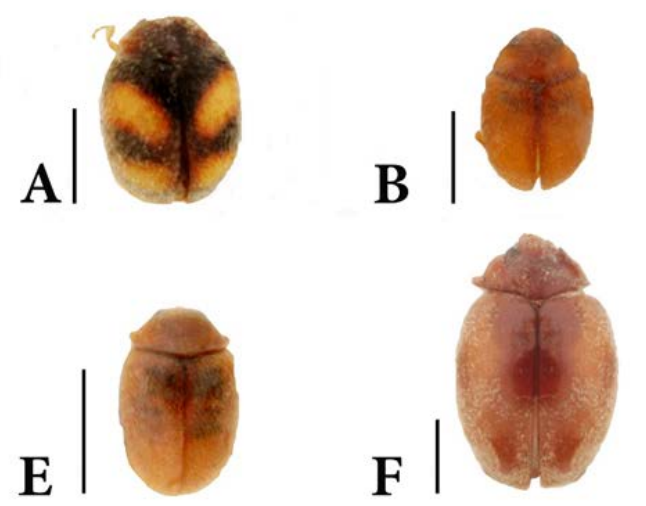

11.9.2001; (3), 20.9.2001; (4), 18.10.2001; (10), 13.3.2002; (4), 3.4.2002; (14), 11.4.2002; (2), 9.5.2002; (1), 6.6.2002; (2), 19.6.2002; (1), 1.8.2002. Shahba, (5), 13.3.2002; (1), 11.4.2002; (1), 11.12.2002. Salkhad, (1), 22.11.2001. Dar`a: Jeelin, (1), 18.7.2002. Zeizon, (1), 21.3.2002; (1), 29.5.2002. Sheikh Miskin, (2), 20.6.2002; (4), 27.5.2003; (10), 22.7.2003. Nahaj, (1), 4.10.2001; (2), 24.10.2001. Fakiia, (1), 28.3.2002. Sahm al-joulan (2), 4.9.2001.

Remarks: Recorded from the Mediterranean Region, Central Europe to North Africa and eastwards to western Asia (Uygun, 1981; Fürsch, 1989; Raimundo and van Harten, 2000). Recorded from Mount Hermon and the Golan Heights (Halperin et al., 1995) and from Wadi Al Qaren (Kabakibi, 1993).

Host species: Amygdalus sp., Cedrus libani, Citrus sp., Cucumis melo, Cupressus sp., Euphorpia sp., Eucalyptus camaldulensis, Ficus carica, Galium sp., Hypericum trinquertifolium, Juglans regia, Ligustrum sp., Malus communis, Medicago sativa, Morus alba, Nerium oleander, Pinus sp., Prunus armeniaca, Prunus spinosa, $P$. mahaleb, Prunus persica, Punica granatum, Pyrus communis, Quercus calliprinos, Robinia pseudoacacia, Rubus idaeus, Spartium junceum, Zea mays, and thistle plants.
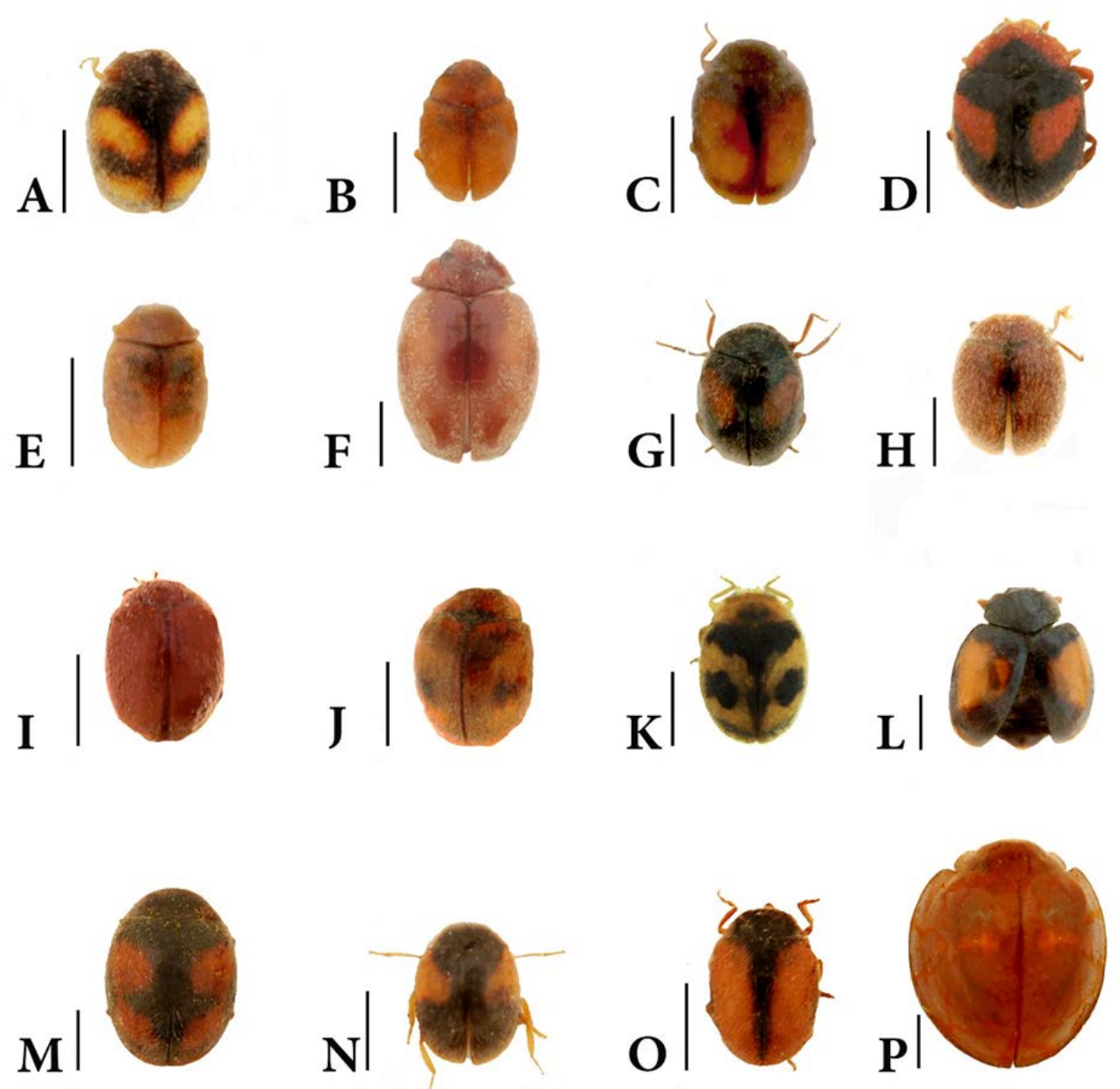

Figure 4: A. Nephus (Sidis) kreissli. B. Scymnus (Mimopullus) pharaonis. C. Scymnus (Mimopullus) flagellisiphonatus. D. Scymnus (Mimopullus) flavicollis. E. Scymnus (Mimopullus) marinus. F. Scymnus (Parapullus) abietis. G. Scymnus (Pullus) apetzi. H. Scymnus (Pullus) auritus. I. Scymnus (Pullus) fraxini. J. Scymnus (Pullus) subvillosus. K. Scymnus (Pullus) syriacus. L. Scymnus (Scymnus) bivulnerus. M. Scymnus (Scymnus) frontalis. N. Scymnus (Scymnus) interruptus. O. Scymnus (Scymnus) nubilus. P. Chilocorus bipustulatus. Scale bar $=1 \mathrm{~mm}$. 


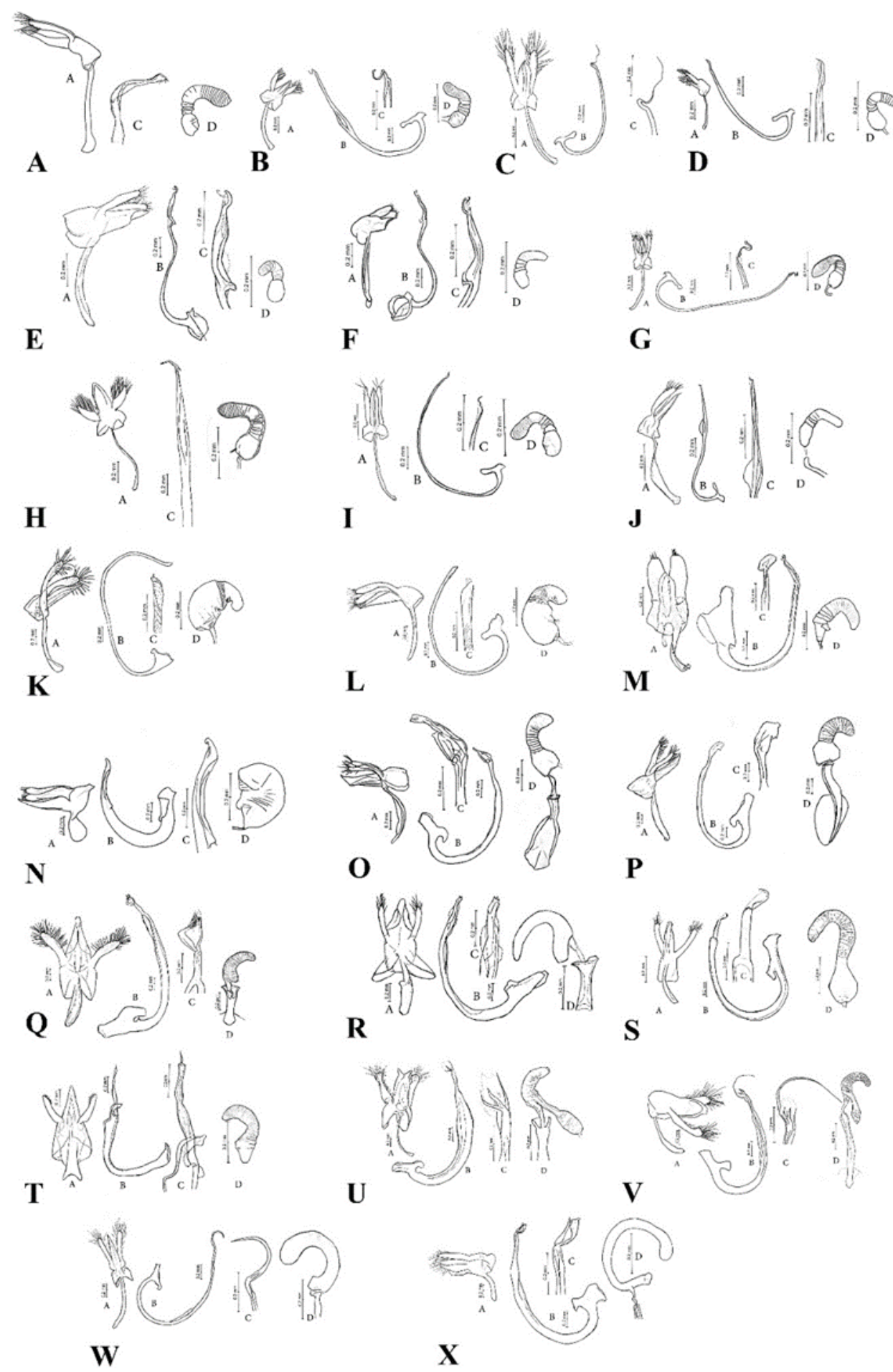

Figure 5: A. Scymnus (Parapullus) abietis. B. Scymnus (Pullus) apetzi. C. Scymnus (Pullus) auritus. D. Scymnus (Pullus) fraxini. E. Scymnus (Pullus) subvillosus. F. Scymnus (Pullus) syriacus. G. Scymnus (Scymnus) bivulnerus. H. Scymnus (Scymnus) frontalis. I. Scymnus (Scymnus) interruptus. J. Scymnus (Scymnus) nubilus. K. Exochomus octosignatus. L. Exochomus quadripustulatus. M. Platynaspis luteorubra. N. Novius cardinalis. O. Adalia (Adalia) bipunctata. P. Adalia (Adalia) decempunctata. Q. Coccinella (Coccinella) septempunctata. R. Coccinella undecimpunctata. S. Harmonia quadripunctata. T. Hippodamia (Adonia) variegata. U. Oenopia conglobata. V. Oenopia oncina. W. Propylea quatuordecimpunctata. X. Psyllobora (Thea) vigintiduopunctata. Key to darwings: A= Tegmen, B= Penis, $\mathrm{C}=$ Penis apex, $\mathrm{D}=$ Spermatheca.

Scymnus (Pullus) syriacus (Marseul, 1868) (Figs. 4K and 5F)

Material examined (250): Damascus: Damascus, (1), 13.5.2003. Damascus Rural: Wadi Al-Qaren, (1), 20.3.2002. Yabrud, (3), 8.8.2002; (1), 11.9.2002. Sasaa, (1), 12.9.2002. Beet-jen Mazraa, (1), 3.10.2001; (1),
8.11.2001; (5), 7.8.2002; (4), 29.8.2002. Erneh, (1), 21.9.2001. Mugr Almeer, (3), 26.8.2003. Addimass, (2), 5.9.2002. Al Hameh, (1), 14.6.2003. Azzabadani, (2), 27.6.2002. Al-Qutayfeh, (9), 15.8.2001; (5), 8.8.2002. Qatana, (3), 14.8.2003. Autaiba, (1), 8.8.2002. Attall, (15), 26.9.2001; (7), 25.10.2001; (7), 26.6.2002; (18), 
10.7.2002; (2), 5.9.2002; (4), 24.8.2003. Kharabo, (1), 22.8.2001; (1), 25.10.2001; (1), 23.5.2002; (1), 11.9.2002; (1), 25.9.2002. Ma`adamiyeh Al-sham, (2), 6.12.2001; (3), 6.3.2002; (1), 12.4.2002; (3), 23.5.2002; (4), 9.9.2002; (2), 4.10.2002; (1), 1.11.2002; (2), 4.12.2002; (1), 5.2.2003; (2), 9.4.2003; (7), 23.6.2003; (27), 11.7.2003; (12), 23.7.2003; (4), 27.7.2003; (10), 13.8.2003. Al-Quneitra: Beer Ajam, (1), 2.10.2002. Masehara, (2), 13.8.2001. Dar`a: Wadi Jeelin, (1), 24.10.2001; (2), 22.11.2001; (1), 7.2.2002; (2), 9.5.2002; (2), 22.8.2002. Zeizon, (1), 8.8.2001; (4), 10.4.2002; (1), 25.4.2002; (1), 9.5.2002; (1), 29.5.2002; (1), 18.7.2002; (2), 18.12.2002; (3), 19.3.2003; (3), 23.4.2003. Sheikh Miskin, (1), 27.5.2003. Sheikh Saad, (1), 13.11.2002. Nahaj, (10), 4.10.2001; (3), 24.10.2001. Sahm al-joulan, (1), 4.9.2001. Namer, (2), 20.6.2002. As`Sweida: Al-Kafr, (1), 29.11.2001; (2), 1.8.2002; (1), 29.11.2002. Ein alarab, (1), 29.11.2001; (1), 9.5.2002; (1), 1.8.2002; (1), 10.9.2002; (1), 23.10.2002. Shahba, (4), 23.3.2002; (5), 11.4.2002; (1), 19.6.2002; (1), 11.12.2002.

Remarks: This species is distributed in the Middle East with reports from Syria, Egypt, Iraq, Turkey and Palestine (Uygun, 1981; Halperin et al., 1995). Listed for Syria by El Hariri (1971). Recorded from northern Syria by Ka'ada (2002). In Iran, it has been recorded as a predator of two mealybugs (Planococcus ficus and Phenacoccus solenopsis) on different hosts (Fallahzadeh et al., 2013).

Host species: Aloysia citrodora, Cedrus libani, Citrus sp., Elaeagnus angustifolia, Ficus carica, Hibiscus esculentus, Inula viscosa, Juglans regia, Morus alba, Nerium oleander, Olea europaea, Pinus sp., Populus sp., Punica granatum, Prunus armeniaca, Prunus avium, Prunus mahaleb, Pyrus communis, Quercus calliprinos, Rubus idaeus, Solanum melongena, Tamarix orticulatus, Vitis vinifera and Zea mays.

Scymnus (Scymnus) bivulnerus Capra and Fürsch, 1967 (Figs. 4L and 5G)

Material examined (52): Damascus Rural: Wadi AlQaren, (1), 5.9.2002; (1), 10.7.2002. Yabrud, (1), 25.10.2001. Ma`adamiyeh Al-sham, (1), 28.6.2002; (1), 30.8.2002; (1), 19.5.2003; (4), 23.6.2003; (5), 11.7.2003; (4), 23.7.2003. Nasseria, (1), 5.6.2002. Sasaa, (2), 3.10.2002; (1), 2.5.2002; (6), 26.8.2003. Beet-jen Mazraa, (2), 26.8.2003. Erneh, (1), 11.7.2002; (1), 29.8.2002. Deir Al-ashairr, (5), 17.10.2001. Azzabadani, (2), 17.10.2001. Attawani, (2), 26.6.2002. As`Sweida: Ein alarab, (2), 17.7.2002. Orman, (1), 22.9.2002; (2), 4.7.2002. Orman Mountain, (1), 21.8.2002; (1), 4.8.2003. Ghoujygoujyat Mountain, (2), 29.4.2003. Qanawat, (1), 6.6.2002.

Remarks: Known from Central and Southern Europe (Gourreau, 1974). Recorded from Palestine (Halperin et al., 1995). Listed by El Hariri (1968) for Syria.

Host species: Amygdalus sp., Citrus sp., Cucurbita pepo, Daucus carota, Eriobotrya japonica, Euphorpia sp., Faba vulgaris, Petrocelinum crispum, Populus sp., Punica granatum, Rubus idaeus, Zea mays and thistle plants.

Scymnus (Scymnus) frontalis (Fabricius, 1787) (Fig. 4M and $5 \mathrm{H}$ )

Material examined (60): Damascus Rural: Kharabo, (4), 22.8.2001; (2), 26.9.2001; (1), 27.11.2001; (2), 23.5.2002; (1), 5.6.2002; (5), 11.7.2002; (1), 24.7.2002;
(26), 11.9.2002; (5), 25.9.2002; (1), 9.10.2002; (1), 25.5.2003; (4), 2.7.2003. Serghaya, (1), 10.7.2002; (1), 24.7.2002; (2), 14.8.2002; (2), 3.10.2002; (1), 7.11.2002.

Remarks: This species is known from Central Asia, West Europe, the Middle East and North Africa (Kuznetsov, 1997). It was listed by El Hariri (1971) in Syria.

Host species: Galium sp., Malus communis, Medicago sativa, Morus alba, Triticum sp., Urtica sp., Zea mays, and thistle plants.

\section{Scymnus (Scymnus) interruptus (Goeze, 1777) (Figs. 4N} and 5I)

Material examined (111): Damascus Rural: Yabrud, (1), 25.10.2001; (2), 26.6.2002. Deir Al-ashairr, (3), 11.6.2002. Al-Hameh, (2), 14.6.2003. Azzabadani, (3), 27.6.2002. Sarkha, (1), 26.6.2002; (1), 11.9.2002. AlQutayfeh, (4), 8.8.2002. Qatana, (4), 5.7.2002. Assaida Zainab, (3), 18.10.2001. Ashiphonieh, (1), 25.9.2002. Attall, (5), 26.9.2001; (1), 25.10.2001; (2), 8.5.2002; (11), 26.6.2002; (24), 10.7.2002. Kharabo, (5), 23.5.2002; (1), 11.7.2002; (2), 25.9.2002; (3), 9.10.2002; (3), 2.4.2003; (6), 25.5.2003; (10), 2.7.2003; (1), 6.8.2003. Serghaya, (1), 10.4.2003. Ma`adamiyeh Al-sham, (1), 28.6.2002; (1), 4.7.2002; (3), 23.6.2003; (5), 11.7.2003; (1), 13.8.2003.

Remarks: Known from Central Europe, North Africa and the Middle East (Canepari, 1991). Recorded from northern Syria by Ka'ada (2002).

Host species: Amygdalus sp., Citrus sp., Cucurbita pepo, Elaeagnus angustifolia, Ficus carica, Galium sp., Juglans regia, Malus communis, Medicago sativa, Morus alba, Phaseolus vulgaris, Populus sp., Punica granatum, Rosa sp., Rubus idaeus, Solanum melongena, Triticum sp., Urtica sp., Zea mays, and thistle plants.

\section{Scymnus (Scymnus) nubilus Mulsant, 1850 (Figs. 40 and $5 \mathrm{~J})$}

Material examined (279): Damascus Rural: Sasaa, (3), 3.10.2002; (1), 7.8.2002; (1), 26.8.2003. Kulaiaa, (2), 7.8.2002. Dorin, (3), 10.7.2002. Zarzar, (1), 14.6.2003. Deir Al-ashairr, (1), 11.6.2002. Qatana, (3), 5.7.2002. Autaiba, (5), 24.7.2002. Assaida Zainab, (8), 18.10.2001; (1), 3.4.2002. Ashiphonieh, (2), 25.9.2002. Attall, (1), 26.9.2001; (6), 26.6.2002; (4), 10.7.2002. Kharabo, (1), 22.8.2001; (2), 25.10.2001; (2), 27.12.2001; (1), 4.5.2002; (2), 15.5.2002; (7), 23.5.2002; (2), 5.6.2002; (15), 11.7.2002; (5), 24.7.2002; (2), 8.8.2002; (3), 11.9.2002; (10), 25.9.2002; (20), 9.10.2002; (1), 29.12.2002; (2), 25.5.2003; (4), 2.7.2003; (1), 6.8.2003. Ma`adamiyeh Alsham, (1), 23.5.2002; (2), 28.6.2002; (2), 9.9.2002; (1), 1.11.2002; (1), 4.12.2002; (2), 19.5.2003; (30), 23.6.2003; (25), 11.7.2003; (15), 23.7.2003; (5), 13.8.2003. AlQuneitra: Jubata Al-Khashab, (1), 16.5.2002. As`Sweida: Ein alarab, (3), 7.8.2001. Ghoujygoujyat Mountain, (1), 4.7.2002. Qanawat, (1), 21.8.2002. Saura, (3), 11.6.2002. Dar`a: Jeelin, (1), 10.8.2001; (1), 18.7.2002. Wadi Jeelin, (1), 29.5.2002; (3), 3.7.2002; (14), 22.8.2002. Zeizon, (1), 9.5.2002; (3), 29.5.2002; (3), 16.6.2003. Izraa, (1), 27.9.2001. Sheikh Miskin, (4), 20.6.2002; (10), 22.7.2003. Sheikh Saad, (2), 12.5.2003. Karak, (1), 22.8.2002. Nahta, (6), 22.8.2002. Nahaj, (1), 24.10.2001. Sehaileia, (1), 20.6.2002. Tafas (2), 25.7.2002. Sahm al-joulan, (3), 4.9.2001. Wadi Shihab, (2), 13.5.2003. Namer, (4), 20.6.2002. 
Remarks: This species have a wide range of distribution in the Mediterranean Region (Raimundo and van Harten, 2000). Listed by El Hariri (1971) and the Golan Heights (Halperin et al., 1995) in Syria. It feeds on aphids and scale insects. In Iran, it has been recorded as a predator of two mealybugs (Nipaecoccus viridis and Maconellicoccus hirsutus) on different hosts (Fallahzadeh et al., 2013).

Host species: Citrus sp., Cucumis sativs, Cucurbita pepo, Cupressus sp., Cydonia vulgaris, Euphorpia sp., Ficus carica, Foeniculum vulgare, Hibiscus esculentus, Juglans regia, Medicago sativa, Morus alba, Nerium oleander, Olea europaea, Pinus sp., Populus sp., Prunus armeniaca, Prunus vulgaris Punica granatum, Quercus calliprinos, Solanum melongena, Triticum sp., Tamarix orticulatus, Urtica sp., Vitis vinifera, Vitex agnus-castus, Zea mays and thistle plants.

\section{Tribe Stethorini}

\section{Stethorus (Stethorus) gilvifrons (Mulsant, 1850)}

Material examined (1368): Al-Quneitra: Beer Ajam, (5), 12.9.2002; (3), 2.10.2002. Jubata Al-Khashab, (2), 10.9.2001; (1), 31.7.2002; (1), 29.8.2002. Hadar, (4), 31.7.2002; (3), 12.9.2002. Masehara, (2), 13.8.2001. Damascus Rural: Attall, (1), 26.9.2001; (7), 25.10.2001; (4), 21.11.2001; (1), 27.12.2001; (3), 14.3.2002 ; (4), 4.4.2002; (1), 8.5.2002; (1), 10.7.2002; (6), 5.9.2002; (7), 20.11.2002; (2), 24.8.2003. Wadi Al-Qaren, (1), 17.11.2001; (3), 5.9.2002. Yabrud, (7), 8.8.2002; (5), 11.9.2002. Sasaa, (4), 13.8.2001; (3), 3.10.2001; (5), 20.3.2002; (7), 2.5.2002; (2), 16.5.2002; (2), 12.6.2002; (1), 7.8.2002; (7), 12.9.2002. Beet-jen Mazraa, (5), 3.10.2001; (1), 7.8.2002; (3), 29.8.2002. Erneh, (7), 21.9.2001; (8), 29.8.2002. Beqaasem, (5), 21.9.2001; (2), 11.7.2002; (1), 29.7.2002. Mugr Almeer, (1), 26.8.2003. Zarzar, (2), 19.9.2001. Deir Al-ashairr, (9), 17.9.2002. Azzabadani, (2), 17.10.2001. Kharabo, (5), 26.9.2001; (8), 25.10.2001; (3), 21.11.2001; (2), 27.12.2001; (6), 23.5.2002; (5), 11.9.2002; (15), 25.9.2002; (18), 9.10.2002; (9), 27.11.2002; (2), 29.12.2002; (3), 9.2.2003; (12), 2.4.2003; (1), 2.7.2003; (2), 6.8.2003. Serghaya, (50), 19.9.2001; (42), 17.10.2001; (9), 7.11.2001; (2), 18.4.2002; (1), 4.5.2002; (1), 10.7.2002; (22), 24.7.2002; (63), 14.8.2002; (39), 5.9.2002; (24), 17.9.2002; (18), 3.10.2002; (16), 7.11.2002; (1), 26.6.2003; (14), 29.7.2003. Ma`adamiyeh Al-sham, (3), 23.5.2002; (46), 30.8.2002; (8), 9.9.2002; (36), 4.10.2002; (30), 1.11.2002; (39), 4.12.2002; (13), 16.1.2003; (8), 5.2.2003; (1), 13.3.2003; (1), 9.4.2003; (1), 23.6.2003. Houch arab (1), 3.9.2001. Assal Alwared, (14), 26.9.2001. Rankus Mountain, (1), 11.9.2002. Sarkha, (8), 26.9.2001; (31), 11.9.2002. Maalula (4), 2.8.2001. Seydnaya, (1), 2.8.2001. Qatana, (2), 14.8.2003. Ahmadia, (1), 25.9.2001. Assaida Zainab, (1), 11.9.2001. Jeesrin, (3), 29.12.2002; (5), 9.2.2003. Ashiphonieh, (51), 25.9.2002. Annashabyeh, (2), 21.11.2001; (1), 24.7.2002. Damascus: Qasioun, (3), 13.5.2003. As`Sweida: Al-Kafr, (8), 20.9.2001. Ein alarab, (28), 7.8.2001; (30), 29.8.2001; (29), 27.9.2001; (6), 18.10.2001; (1), 1.8.2002; (17), 21.8.2002; (48), 10.9.2002; (20), 23.10.2002. Orman Mountain, (18), 1.8.2001; (23), 12.8.2001; (20), 29.8.2001; (15), 27.8.2001; (1), 11.4.2002; (1), 1.8.2002; (49), 10.9.2002; (1), 4.8.2003. Orman, (20), 29.8.2001; (6), 4.7.2002; (6),
17.7.2002. Taamri Mountain, (2), 17.7.2002; (1), 21.8.2002(2), 5.8.2003; (1), 8.8.2003. Ghoujygoujyat Mountain, (16), 1.8.2002; (33), 21.8.2002; (10), 10.9.2002; (10), 23.10.2002; (7), 22.10.2003. Qanawat, (1), 12.8.2001; (3), 11.9.2001; (1), 20.9.2001. Taima, (4), 11.9.2001. Tal-loz, (2), 12.8.2001. Mashkok, (1), 20.9.2001. Dar`a: Wadi Jeelin, (5), 21.9.2001; (3), 22.11.2001; (4), 7.2.2002; (3), 25.4.2002. Jeelin, (3), 24.10.2001; (5), 3.7.2002. Zeizon, (4), 8.8.2001; (1), 22.8.2002. Mzireeb, (1), 6.8.2001. Sheikh Saad, (2), 13.11.2002. Nahaj, (18), 4.10.2001; (16), 24.10.2001. Sahm al-joulan, (3), 4.9.2001.

Remarks: Distributed in the East Mediterranean Region, covering India, Pakistan, Afghanistan, the Cuacasues, Turkey, Iran and Saudi Arabia (Gourreau, 1974; Uygun, 1981; Raimundo and van Harten, 2000).

Host species: Aloysia citrodora, Amygdalus sp., Capsicum annum, Citrus sp., Cucurbita pepo, Cupressus sp., Eriobotrya japonica, Eucalyptus camaldulensis, Ficus carica, Glycyrrhiza glabra, Helianthus annus, Hibiscus esculentus, Inula viscosa, Juglans regia, Ligustrum sp., Lonicera sp., Malus communis, Medicago sativa, Morus alba, Olea europaea, Parietaria judaicus, Phaseolus vulgaris, Pinus sp., Populus sp., Prunus armeniaca, Pyrus communis, Pistacia vera, Prunus avium, Prunus mahaleb, Prunus vulgaris, Punica granatum, Quercus calliprinos, Rosa sp., Rubus idaeus, Schinus molle, Solanum melongena, Urtica sp., Vitis vinifera, and Zea mays.

Tribe Chilocorini Mulsant, 1846 Chilocorus bipustulatus (Linnaeus, 1758) (Fig. 4P)

Material examined (42): Al-Quneitra: Jubata AlKhashab (1), 29.8.2002. Damascus Rural: Ma`adamiyeh Al-Sham, (9) 9.9.2002; (15) 13.8.2003; (4) 24.8.2003; (3) 15.8.2004. Addimass, (1), 17.9.2002; (1), 3.4.2003. Airport Street (1), 28.8.2001. Attall (1), 15.8.2002. As`Sweida: Ein alarab, (1), 1.8.2002; (2), 10.9.2002; (1), 23.10.2002. Dar`a: Wadi Jeelin, (1), 7.2.2002; (1), 29.5.2002.

Remarks: This species has a wide range of distribution covering India, Pakistan, China, Mongolia, Russia and Western Europe (Poorani, 2004), with records from northern Syria and the coastal regions (Lababidi and Zepter, 1995; Al Jundi and Ahmad, 1999) and Damascus (Bascheer and Abo Alshamat, 2004). It feeds on scale insects and aphids. Lababidi and Zebitz (1995) reported it feeding on the pistachio psyllid Agonoscena targionii Lich. in northern Syria.

Host species: Biota orientalis, Citrus sp., Cupressus sp., Olea europaea, Pinus sp., Populus sp., Pyrus communis, and Quercus calliprinos,

Exochomus octosignatus (Gebler, 1830) (Figs. 5K and 6A)

Material examined (1): Damascus Rural: Bludan Mountain, (1), 14.6.2003.

Remarks: This species is recorded for the first time in Syria. It was listed under the genus Brumus. Distribution of this species extends from Siberia to the Eastern Mediterranean (Duverger, 1991). Uygun (1981) recorded this species to feed on scale insects in Turkey.

Host species: Species of family Asteraceae. 

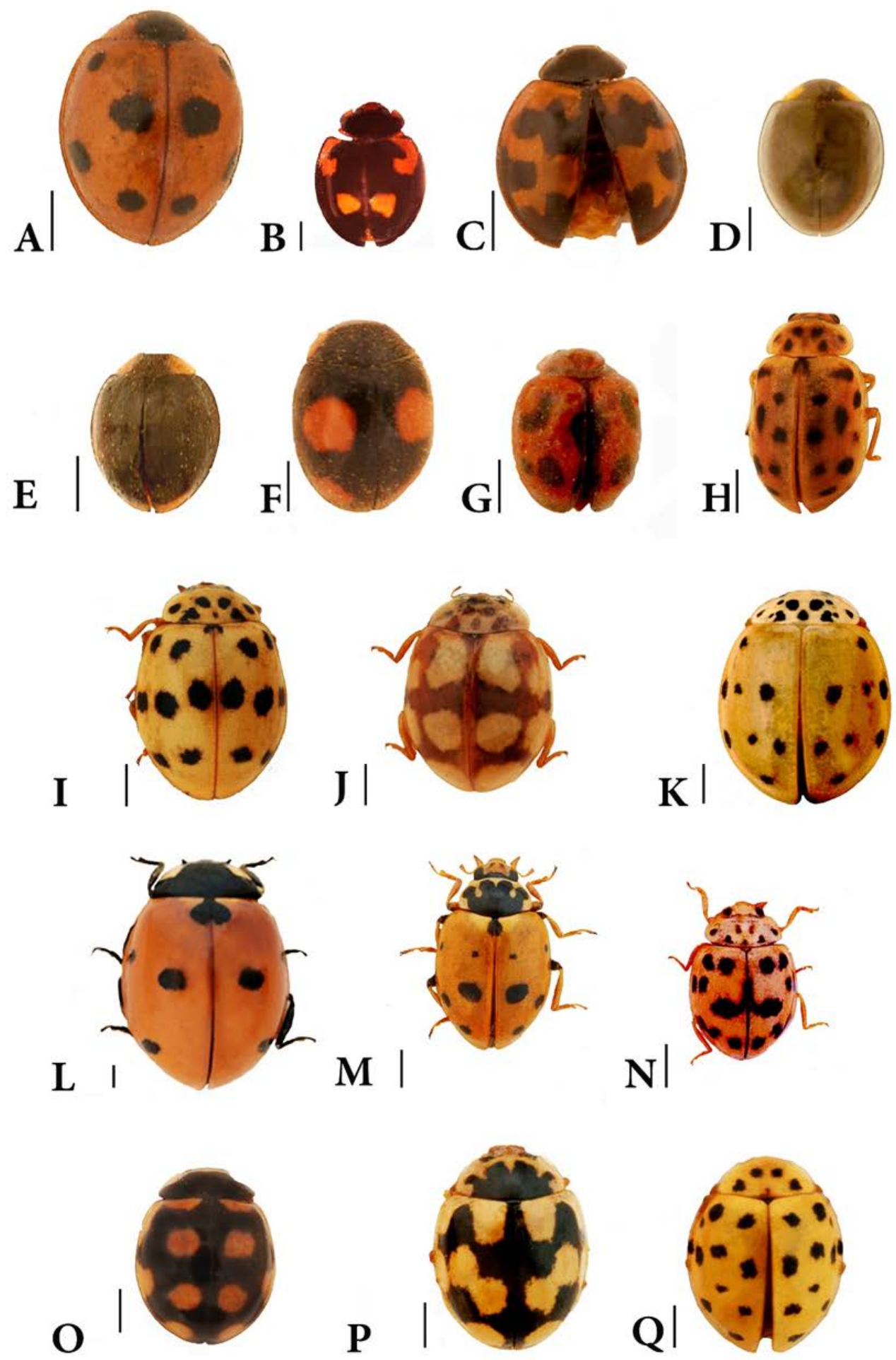

Figure 6: A. Exochomus octosignatus. B. Exochomus quadripustulatus. C. Exochomus undulatus. D. Parexochomus nigromaculatus. E. Parexochomus pubescens. F. Platynaspis luteorubra. G. Novius cardinalis. H. Anisosticta novemdecimpunctata. I. Adalia (Adalia) bipunctata. J. Adalia (Adalia) decempunctata. K. Harmonia quadripunctata. L. Coccinella (Coccinella) septempunctata. M. Hippodamia (Adonia) variegata. N. Oenopia conglobata. O. Oenopia oncina. P. Propylea quatuordecimpunctata. Q. Psyllobora (Thea) vigintiduopunctata. Scale bar $=1 \mathrm{~mm}$.

\section{Exochomus quadripustulatus (Linnaeus, 1758) (Figs. 5L and 6B)}

Material examined (54): Damascus Rural: Wadi AlQaren, (4), 19.8.2001; (1), 17.11.2001; (1), 14.3.2002; (2), 19.2.2003. Al-Quneitra: Beer Ajam, (2), 20.3.2002; (3), 19.3.2003. Jubata Al-Khashab, (2), 28.2.2002; (3), 25.4.2002; (14), 16.5.2002; (10), 16.4.2003; (1),
26.8.2003; (4), 2.5.2005. As`Sweida: Al-Kafr, (4), 23.10.2002; (1), 12.12.2002; (2), 11.5.2003.

Remarks: This species is distributed from India, Mangolia, Middle East to Russian Far East and Western Europe (Kuznetsov, 1997; Poorani, 2004; Kováŕ, 2007). In Syria, it was listed by El Hariri (1968) and recorded from Wadi Al Qaren by Kabakibi (1993), from As`Sweida by 
Almatni et al. (1999) and from Damascus by Bascheer and Abo Alshamat (2004).

Host species: Amygdalus sp. and Quercus calliprinos.

\section{Exochomus undulatus Weise, 1878 (Fig. 6C)}

Material examined (2): Damascus Rural: Rankus, (1), 6.7.2004. Wadi Al-Qaren, (1), 14.3.2002.

Remarks: This species is known from the southern states of the former Soviet Union, Turkey, Iran, Palestine and Egypt (Alfieri, 1976; Uygun, 1981, Moodi, 1994; Halperin et al., 1995). In Syria, it was listed by El Hariri (1968). Uygun (1981) recorded it feeding on scale insects and aphids in Turkey. Jalilvand et al. (2014) found that this species predates on Planococcus vovae in Iran..

Host species: Amygdalus sp. and Quercus calliprinos.

\section{Parexochomus nigromaculatus (Goeze, 1777) (Fig. 6D)}

Material examined (24): Damascus Rural: Wadi AlQaren, (1), 19.9.2001; (2), 5.9.2002. Jeesrin, (1), 29.12.2001. Al-Quneitra: Masehara, (1), 13.8.2001. As`Sweida: Al-Kafr (1), 18.10.2001. Taamri Mountain, (1), 21.6.2002. Orman Mountain, (1), 21.8.2002; (1), 8.8.2003. Qanawat, (2), 12.8.2001. Dar`a: Izraa (4), 27.9.2001. Sheikh Miskin, (8), 30.8.2001. Sanameen, (1), 6.8.2001.

Remarks: This species has a wide range of distribution in South and Central Europe, Africa and Asia (Raimundo and van Harten 2000). This species was recorded previously from Syria by El Hariri (1971) and from the Golan Heights (Halperin et al., 1995). Jalilvand et al. (2014) found that this species predates on Phenacoccus sp. in Iran.

Host species: Achillea sp., Cucumis melo, Cucurbita pepo, Daucus carota, Nerium oleander, Quercus calliprinos and Urtica sp.

\section{Parexochomus pubescens (Küster, 1848) (Fig. 6E)}

Material examined (6): Damascus Rural: Jeesrin, (3), 9.2.2003. Attall, (1), 24.8.2003. Kharabo, (1), 23.5.2002. Dar`a: Wadi Shihab, (1), 13.5.2003.

Remarks: This is widely distributed species with a range extending from India, Middle East, reaching westwards to Spain, with records from Egypt, Palestine, Syria, Saudi Arabia and Yemen (Raimundo and van Harten, 2000; Poorani, 2004).

Host species: Tamarix articulata, Triticum sp. and Urtica sp.

\section{Tribe Platynaspidini Mulsant, 1846}

Platynaspis luteorubra (Goeze, 1777) (Figs. 5M and 6F)

Material examined (14): Damascus Rural: Kharabo, (1), 22.8.2001; (1), 11.7.2002; (1), 24.7.2002; (1), 6.8.2003. Qatana, (1), 5.7.2002. Attall, (1), 10.7.2002. As`Sweida: Ein alarab (1), 22.7.2003. Orman Mountain (1), 27.9.2001. Ghoujygoujyat Mountain, (1), 4.7.2002; (2), 29.4.2003. Shahba, (1), 13.3.2002. Dar`a: Zeizon, (2), 8.8.2001.

Remarks: Halperin et al. (1995) reported Platynaspis luteorubra as a rare species in the Golan Heights and Palestine. This is a Palaearctic species with a range extending from North Africa, Europe, and the Middle East reaching the Caucasues (Uygun, 1981).
Host species: Malus communis, Medicago sativa, Morus alba, Pinus sp., Punica granatum, Rosa sp., and Zea mays.

Tribe Noviini Mulsant, 1850

Novius cardinalis (Mulsant, 1850) (Figs. 5N and 6G)

Material examined (16): Damascus Rural: Attall, (1), 25.10.2001; (1), 27.12.2001. Ma`adamiyeh Al-sham, (1), 15.2.2002; (1), 23.5.2002; (1), 16.1.2003; (1), 5.2.2003; (1), 13.3.2003; (1), 9.4.2003; (1), 23.6.2003; (1), 11.7.2003; (1), 13.8.2003. Dar`a: Wadi Jeelin, (1), 7.2.2002; (2), 25.4.2002. Zeizon, (1), 25.4.2002; (1), 27.5.2002.

Remarks: This species has a world-wide distribution (Uygun, 1981). Listed previously from Syria (El Hariri, 1968). Recorded by Al Jundi and Ahmad (1999) from the coastal regions.

Host species: Citrus sp., Faba vulgaris, Punica granatum, and Rubus idaeus.

\section{Tribe Tytthaspidini Crotch, 1874}

Anisosticta novemdecimpunctata (Linnaeus, 1758) (Fig. 6H)

Material examined (1): Dar`a: Nahta, (1), 22.8.2002.

Remarks: This species is widely distributed in Europe, Central Asia and the Middle East (Uygun, 1981; Kuznetsov, 1997; Kováŕ, 2007). This species is recorded for the first time in Syria.

Host species: Zea mays.

Tribe Coccinellini Latreille, 1807

Adalia (Adalia) bipunctata (Linnaeus, 1758) (Figs. 50 and 6I)

Material examined (61): Damascus Rural: KafrHawar, (1), 7.8.2002. Al-Hameh, (2), 14.6.2003. Jeesrin, (30), 29.12.2002; (3), 9.2.2003. Attall, (3), 26.6.2002. Kharabo, (2), 22.8.2001; (1), 25.10.2001; (1), 23.5.2002; (1), 11.7.2002; (1), 24.7.2002; (1), 8.8.2002; (3), 11.9.2002; (1), 25.9.2002; (3), 9.10.2002; (2), 2.4.2003; (2), 25.5.2003; (1), 2.7.2003; (1), 6.8.2003. Ma`adamiyeh Al-Sham, (1), 23.5.2002. As`Sweida: Orman Mountain, (1), no date.

Remarks: This species has a world-wide distribution (Harde, 1999, Poorani, 2004). In Syria, it was listed by El Hariri (1968). Recorded from Damascus by Bascheer and Abo Alshamat (2004) and from As`Sweida by Almatni et al. (1999).

Host species: Ficus carica, Juglans regia, Malus communis, Medicago sativa, Morus alba, Populus sp., Punica granatum, Rubus idaeus, Salix sp., Solanum melongena, Triticum sp., Urtica sp., and Zea mays.

Adalia (Adalia) decempunctata (Linnaeus, 1758) (Figs. $5 P$ and $6 \mathrm{~J}$ )

Material examined (37): Al-Quneitra: Beer Ajam, (2), 27.6.2002; (1), 2.10.2002. Damascus Rural: Wadi Al-Qaren, (4), 10.7.2002. Sarkha, (5), 26.6.2002; (1), 11.9.2002. Beqaasem, (1), 11.7.2002. Kafr Hawar, (1), 7.8.2002. As`Sweida: Al-Kafr, (1), 17.7.2002; (2), 23.10.2002. Orman, (1), 24.6.2002; (3), 11.7.2002; (1), 17.7.2002. Taamri Mountain, (1), 4.7.2003. Ghoujygoujyat Mountain, (1), 4.7.2003. Qanawat, (2), 6.6.2002. Sad alaain, (9), 17.7.2002. Dar`a: Zeizon, (1), 9.5.2002.

Remarks: It is distributed throughout the Palaearctic region, North Africa reaching Japan (Harde, 1999; Uygun, 
1981). Previous records from Syria include the coastal region (Al Jundi and Ahamd, 1999), Mount Hermon (Halperin et al., 1995), North Syria (Aswad, 1998) and listed by El Hariri (1968).

Host species: Amygdalus sp., Inula viscosa, Juglans regia, Quercus calliprinos, Robinia pseudoacacia, Salix sp., Pinus sp., Populus sp., and Prunus vulgaris.

Coccinella (Coccinella) septempunctata Linnaeus, 1758 (Figs. 5Q and 6L)

Material examined (330): Al-Quneitra: Beer Ajam, (1), 27.6.2002. Jubata Al-Khashab, (12), 12.6.2002; (8), 11.7.2002; (3), 31.7.2002, (2), 26.8.2003. Damascus: Qasioun, (6), 13.5.2003. Damascus Rural: Kharabo, (1), 22.8.2001; (1), 21.11.2001; (1), 6.2.2002; (2), 18.4.2002; (8), 15.5.2002; (9), 23.5.2002; (1), 5.6.2002; (1), 24.7.2002; (1), 11.9.2002; (1), 2.4.2003; (1), 21.4.2003; (2), 13.5.2003; (3), 25.5.2003; (1), 6.8.2003; (6) 13.5.2005. Yabrud, (1), 25.10.2001; (1), 4.4.2002; (4), 8.5.2002. Sasaa, (1), 2.5.2002; (10), 16.5.2002. Beet-jen Mazraa, (6), 8.11.2001; (5), 16.5.2002. Qatana, (1), 5.7.2002; (1), 28.2.2002. Deir Al-ashairr, (2), 11.6.2002. Bludan, (2), 14.6.2003. Rankaus, (1), 10.6.2003. Maalula, (1), 8.5.2002. Attawani, (1), 20.11.2002. Autaiba, (6), 6.2.2002; (1), 9.2.2003. Deir Atyeh, (4), 21.5.2003. Annashabyeh, (1), 28.8.2001. Attall, (3), 3.9.2001; (6), 25.10.2001; (3), 27.12.2001; (2), 14.3.2002; (1), 4.4.2002; (2), 20.11.2002; (6), 19.2.2003; (1), 7.5.2003. Serghaya, (4), 19.9.2001; (10), 7.11.2001; (2), 6.12.2001; (7), 4.5.2002; (8), 30.5.2002; (12), 11.6.2002; (2), 27.6.2002; (1), 17.9.2002; (4), 3.10.2002; (4), 7.11.2002; (2), 22.5.2003. Ma`adamiyeh Al-sham, (1), 24.11.2001; (2), 19.4.2002; (2), 24.4.2002; (1), 1.5.2002; (1), 23.5.2002; (1), 19.7.2002; (1), 1.11.2002; (1), 4.12.2002; (3), 19.5.2003; (1), 23.6.2003; (1), 23.7.2003. As`Sweida: AlKafr, (3), 12.6.2003. Ein alarab, (2), 9.5.2002; (16), 6.6.2002; (1), 19.6.2002; (1), 23.10.2002; (5), 2.6.2003. Orman Mountain, (3), 11.4.2002; (1), 6.6.2002; (2), 12.6.2003. Taamri Mountain, (2), 17.4.2002; (1), 17.7.2002. Ghoujygoujyat Mountain, (2), 4.7.2002; (1), 12.6.2003. Qanawat, (1), 29.11.2001; (1), 13.3.2002 (1), 11.4.2002; (1), 9.5.2002; (1), 6.6.2002. Shahba, (2), 29.11.2001; (4), 13.3.2002; (1), 11.4.2002; (3), 11.5.2003. Tarba, (1), 11.9.2001. Saura, (1), 13.3.2002. Dar`a: Wadi Jeelin, (1), 9.5.2002. Zeizon, (6), 22.11.2001; (4), 7.2.2002; (1), 10.4.2002; (1), 25.4.2002; (1), 29.5.2002; (1), 18.12.2002; (1), 16.6.2003. Mzireeb, (1), 20.12.2001; (1), 25.4.2002; (4), 9.5.2002; (3), 16.5.2002; (1), 18.12.2002. Sheikh Saad, (8), 12.5.2003. Ajami, (3), 13.11.2002. Sehaileia, (1), 20.6.2002. Tafas (1), 22.11.2001; (4), 25.7.2002. Dar`a, (3), 6.5.2003. Wadi Shihab, (2), 13.5.2003. Namer, (10), 15.4.2002.

Remarks: This species has a world-wide distribution (Zhou et al., 1995). In Syria, it was listed by El Hariri (1968) and collected from Wadi Al Qaren by Kabakibi (1993), from As`Sweida by Almatni et al. (1999) and from Damascus by Bascheer and Abo Alshamat (2004). It feeds on aphids. Lababidi and Zebitz (1995) reported it feeding on the pistachio psyllid Agonoscena targionii Lich. in northern Syria.

Host species: Acer sp., Achillea sp., Amygdalus sp., Anthemis sp., Beta vulgaris, Cardus pycnocephalus, Centaurea sp., Chenopodium sp., Citrus sp. Cucumis sativs, Cucurbita pepo, Cupressus sp., Diplotaxis ercoides,
Elaeagnus angustifolia, Faba vulgaris, Foeniculum vulgare, Hibiscus esculentus, Hordeum sp., Inula viscosa, Juglans regia, Malus communis, Medicago sativa, Morus alba, Nerium oleander, Onobryclus sp., Ononis spinosa, Onopordum syriacum, Quercus calliprinos, Petroselinum crispum, Pinus sp., Punica granatum, Prunus avium, Prunus armeniaca, Prunus vulgaris, Pyrus communis, Rosa sp., Rubus idaeus, Tamarix orticulatus, Triticum sp., Urtica sp., Vicia sativa, Vitis vinifera, and Zea mays.

Coccinella (Spilota) undecimpunctata aegyptica Reiche, 1861 (Figs. 5R)

Material examined (152): Damascus: Damascus, (1), 16.6.2002; (1), 13.5.2003. Damascus Rural: Kharabo, (2), 22.8.2001; (2), 25.10.2001; (2), 15.5.2002; (4), 23.5.2002; (2), 5.6.2002; (2), 11.7.2002; (1), 13.5.2003; (1), 25.5.2003. Serghaya, (1), 27.6.2002. Ma`adamiyeh AlSham, (8), 24.4.2002; (5), 23.5.2002; (4), 19.5.2003; (1), 11.7.2003. Yabrud, (5), 8.5.2002. Nasseria, (2), 5.6.2002. Kanaker, (3), 4.9.2001. Zarzar, (1), 14.6.2003. Deir Alashairr, (1), 17.10.2001. Bludan, (2), 14.6.2003. Assal Alwared, (1), 26.9.2001. Rankus Mountain, (1), 21.5.2003. Rankaus, (6), 10.6.2003. Al-Dumayr, (15), 15.8.2001. Qatana, (1), 5.7.2002. Seydnaya, (5), 21.5.2003. Ahmadia, (10), 23.5.2002. Autaiba, (8), 10.7.2001; (1), 28.8.2001; (6), 2.8.2004. Assaida Zainab, (2), 11.9.2001. Deir Atyeh, (1), 21.5.2003. Annashabyeh, (14), 28.8.2001. Attall, (1), 3.9.2001; (1), 7.5.2003. As`Sweida: Al-Kafr, (1), 12.6.2003. Ein alarab, (1), 2.6.2003. Orman Mountain, (1), 23.6.2002; (1), 16.5.200. Ghoujygoujyat Mountain, (1), 4.7.2002. Taamri Mountain, (1), 6.6.2003; (1), 25.7.2003. Orman, (1), 6.6.2003. Qanawat, (2), 29.11.2001. Sad alaain, (1), 12.5.2003. Dar`a: Jeelin, (2), 10.8.2001. Zeizon, (1), 24.10.2001. Sheikh Miskin, (1), 27.5.2003. Mzireeb, (2), 6.8.2001; (1), 20.12.2001. Sheikh Saad, (1), 12.5.2003. Shagara, (1), 9.7.2002. Tafas, (3), 25.7.2002. Daraa, (3), 6.5.2003. Shihab, (1), 25.7.2002. Namer, (1), 15.4.2002.

Remarks: Known from Europe, Asia and Africa (Rainundo and van Hartenl 2000). In Syria, recoded by Al Jundi and Ahmad (1999) as a predator for aphids on citrus tree in the coastal regions and recorded from As 'Sweida by Almatni et al. (1999). It was listed by El Hariri (1968) from Syria.

Host species: Amygdalus sp., Beta vulgaris, Carthamus tinctorius, Cerasus avium, Citrus sp., Crataegus sp., Cucumis sativus, Cupressus sp., Eriobotrya japonica, Euphorpia sp., Faba vulgaris, Foeniculum vulgare, Gossypium sp., Hordeum sp., Juglans regia, Malus communis, Morus alba, Medicago sativa, Nerium oleander, Pinus sp., Pisum sativum, Prunus armeniaca, Punica granatum, Pyrus communis, Solanum melongena, Triticum sp., Vicia sativa and Zea mays.

Harmonia quadripunctata (Pontoppidan, 1763) (Figs. 5S and 6K)

Material examined (59): Damascus Rural: Zarzar, (1), 30.4.2003; (1), 14.6.2003. Maissalon, (4), 30.4.2003. Addimass, (1), 15.5.2002; (2), 30.4.2003. Kafr Hawar, (1), 7.8.2002. Al-Quneitra: Trounja 7: (1), 28.2.2002; (5), 29.8.2002; (1), 16.4.2003; (1), 12.6.2002. As`Sweida: AlKafr, (1), 23.10.2002, Ein alarab, (7), 4.7.2002; (3), 17.7.2002; (9), 1.8.2002; (8), 21.8.2002; (2), 10.9.2002; (3), 23.10.2002; (3), 2.6.2003. Ghoujygoujyat Mountain, 
(2), 1.8.2002; (1), 23.10.2002. Taamri Mountain, (1), 25.7.2003. Orman Mountain, (1), 4.8.2003.

Remarks: Known from Europe throughout Central Asia and the Middle East (Allawi, 1989; Canepari, 1991; Halperin et al., 1995; Harde, 1999). It was recoded by Al Jundi and Ahmad (1999) as a predator for aphids on citrus tree in the coastal regions.

Host species: Amygdalus sp., Cedrus libani, Cupressus sp., Galium sp., Pinus sp., Prunus avium, Prunus vulgaris, Quercus calliprinos and Rosa damascena. Hippodamia (Adonia) variegata (Goeze, 1777) (Figs. 5T
and 6M)

Material examined (1042): Damascus Rural: Attall, (25), 3.9.2001; (4), 26.9.2001; (2), 27.12.2001; (4), 4.4.2002; (2), 8.5.2002; (1), 26.6.2002; (11), 10.7.2002; (3), 20.11.2002; (7), 7.5.2002. Kharabo, (1), 22.8.2001; (1), 26.9.2001; (6), 25.10.2001; (3), 21.11.2001; (1), 6.2.2002; (5), 4.5.2002; (11), 15.5.2002; (102), 23.5.2002; (6), 5.6.2002; (11), 11.7.2002; (6), 24.7.2002; (1), 8.8.2002; (24), 11.9.2002; (5), 25.9.2002; (3), 9.10.2002; (3), 25.5.2003; (6), 2.7.2003; (8), 6.8.2003; (2), 13.5.2005. Serghaya, (7), 19.9.2001; (19), 17.10.2001; (25), 7.11.2001; (2), 6.12.2001; (1), 18.4.2002; (2), 30.5.2002; (17), 11.6.2002; (16), 27.6.2002; (14), 10.7.2002; (11), 24.7.2002; (15), 14.8.2002; (2), 5.9.2002; (6), 17.9.2002; (8), 3.10.2002; (15), 7.11.2002; (2), 9.1.2003; (1), 10.4.2003; (2), 26.6.2003; (3), 29.7.2003. Ma`adamiyeh Al-sham, (1), 6.12.2001; (1), 20.1.2002; (12), 19.4.2002; (2), 1.5.2002; (15), 23.5.2002; (6), 28.6.2002; (2), 19.7.2002; (1), 9.9.2002; (2), 16.1.2003; (4), 5.2.2003; (3), 13.3.2003; (8), 19.5.2003; (4), 23.6.2003; (7), 11.7.2003; (2), 23.7.2003; (1), 13.8.2003. Yabrud 14: (4), 2.8.2001; (1), 26.9.2001; (8), 25.10.2001; (1), 11.9.2002. Kanaker, (1), 4.9.2001. Sasaa, (13), 3.10.2001; (1), 12.6.2002. Kulaiaa, (4), 7.8.2002. Dorin, (12), 10.7.2002; (5), 24.8.2003. Beet-jen Mazraa 4: (1), 1.8.2001; (3), 8.11.2001. Erneh (4), 11.7.2002; Zarzar, (7), 19.9.2001; (1), 14.6.2003. Deir Al-ashairr, (12), 17.10.2001; (8), 28.11.2001; (17), 11.6.2002. Al-Hameh, (5), 14.6.2003. Azzabadani, (3), 17.10.2001; (1), 28.11.2001; (6), 27.6.2002. Bludan, (4), 14.6.2003. Houch arab, (2), 3.9.2001. Rankaus, (6), 21.5.2003; (10), 10.6.2003. Attawani, (1), 20.11.2002. Al-Dumayr, (1), 15.8.2001. Qatana, (19), 5.7.2002. Ahmadia, (3), 25.9.2002. Airport Street, (2), 21.11.2001. Autaiba, (1), 6.2.2002; (1), 24.7.2002. Haran Al-awameed, (1), 24.7.2002. Assaida Zainab, (9), 11.9.2001; (1), 3.4.2002. Deir Atyeh, (1), 21.5.2003. Jeesrin, (21), 29.12.2002; (1), 9.2.2003. Ashiphonieh, (6), 25.9.2002. Annashabyeh, (10), 28.8.2001. Damascus: Damascus, (9), 13.5.2003. As`Sweida: Al-Kafr, (1), 1.8.2002; (1), 12.6.2003. Ein alarab, (1), 21.11.2001; (2), 29.11.2001; (1), 19.6.2002; (5), 17.7.2002; (8), 1.8.2002; (1), 23.10.2002; (2), 2.6.2003. Orman Mountain, (1), 21.11.2001; (4), 4.7.2002; (14), 27.9.2002; (1), 1.8.2002; (2), 21.8.2002; (1), 12.6.2003. Taamri Mountain, (2), 17.4.2002; (3), 6.6.2003; (1), 13.6.2003; (1), 28.6.2003; (1), 4.7.2003; (2), 25.7.2003. Orman, (15), 21.6.2002; (6), 4.7.2002; (1), 17.7.2002; (4), 22.6.2002; (1), 11.7.2002; (4), 25.5.2003; (1), 6.6.2003. Ghoujygoujyat Mountain, (3), 10.9.2002; (1), 21.8.2002; (4), 23.10.2002; (2), 29.4.2003; (1), 12.6.2003. Sad alaain, (6), 17.7.2002. Qanawat, (9), 12.8.2001; (3), 29.8.2001; (1), 11.9.2001; (8), 18.10.2001;
(10), 15.5.2002; (1), 19.6.2002; (5), 1.8.2002; (8), 21.8.2002; (5), 4.8.2005; Dar`a: Shahba, (1), 16.5.2001; (1), 13.3.2002; (1), 11.12.2002; (1), 11.5.2003. Jeelin, (15), 10.8.2001; (3), 30.8.2001; (6), 18.7.2002. Wadi Jeelin, (2), 21.9.2001; (1), 22.11.2002; (1), 25.4.2002; (4), 9.5.2002; (1), 2.10.2002. Zeizon, (9), 8.8.2001; (3), 24.10.2001; (18), 22.11.2001; (3), 20.12.2001; (5), 10.4.2002; (1), 9.5.2002; (2), 29.5.2002; (1), 16.6.2003. Izraa, (6), 27.9.2001. Sheikh Miskin, (6), 30.8.2001; (1), 20.6.2002; (2), 27.5.2003; (4), 22.7.2003. Mzireeb, (4), 6.8.2001; (7), 20.12.2001; (2), 25.4.2002; (4), 9.5.2002; (3), 16.5.2002; (1), 25.7.2002. Sheikh Saad, (1), 12.5.2003. Ajami, (2), 13.11.2002. Nahta, 2: (1), 6.8.2002; (1), 22.8.2002. Sehaileia, (1), 20.6.2002. Tafas 23: (2), 4.9.2001; (13), 22.11.2001; (8), 25.7.2002. Dar`a, (6), 6.5.2003. Shihab, (1), 25.7.2002. Wadi Shihab, (1), 13.5.2003. Namer, (1), 20.6.2002.

Remarks: This species has a wide range of distribution extending from the Russian Far East across Asia southward to India and the Middle East to Europe and North Africa and occurs also in South Africa (Kuznetsov, 1997; Raimundo and van Harten, 2000). Listed from Syria by El Hariri (1968). Recorded by Al Jundi and Ahmad (1999) from the coastal regions. Its principal food is aphids. Özden et al. (2006) reported this species from Cyprus feeding on aphids and mites. Lababidi and Zebitz (1995) reported it feeding on the pistachio psyllid Agonoscena targionii Lich. in northern Syria.

Host species: Acer sp., Agropyron repens, Amygdalus sp., Anabasis sp., Beta vulgaris, Carthamus tinctorius, Cedrus libani, Citrus sp., Crataegus sp., Cucurbita pepo, Cucumis melo, Cupressus sp., Diplotaxis ercoides, Elaeagnus angustifolia, Eriobotrya japonica, Euphorpia sp., Faba vulgaris, Ferula hermonis, Ficus carica, Galium sp., Glycyrrhiza glabra, Helianthus innuus, Hibiscus esculentus, Hordeum sp., Hypericum trinquertifolium, Inula viscosa, Juglans regia, Malus communis, Malva sp., Medicago sativa, Nerium oleander, Ocimum sp., Petrocelinum crispum, Papaver syriacum, Pinus sp., Pistacia vera, Populus sp., Punica granatum, Prunus mahaleb, Prunus vulgaris, Pyrus communis, Quercus calliprinos, Rosa sp., Rubus idaeus, Sinapis alba, Solanum melongena, Spartium junceum, Triticum sp., Urtica sp., and Zea mays.

\section{Oenopia conglobata (Linnaeus, 1758) (Figs. 5U and 6N)}

Material examined (618): Damascus Rural: Attall, (3), 3.9.2001; (3), 25.10.2001; (1), 14.3.2002; (1), 8.5.2002; (3), 26.6.2002; (3), 10.7.2002; (2), 7.5.2003. Kharabo, (14), 22.8.2001; (1), 26.9.2001; (5), 25.10.2001; (1), 21.11.2001; (4), 23.5.2002; (1), 5.6.2002; (1), 11.7.2002; (3), 24.7.2002; (9), 8.8.2002; (3), 11.9.2002; (1), 25.9.2002; (4), 9.10.2002; (1), 29.12.2002; (1), 2.4.2003; (5), 13.5.2003; (7), 25.5.2003; (9), 6.8.2003; (1), 9.5.2002. Serghaya, (2), 19.9.2001; (5), 17.10.2001; (3), 7.11.2001; (1), 18.4.2002; (4), 4.5.2002; (5), 15.5.2002; (3), 30.5.2002; (1), 11.6.2002; (14), 5.9.2002; (23), 17.9.2002; (22), 3.10.2002; (17), 7.11.2002; (17), 10.4.2003; (6), 22.5.2003; (3), 29.7.2003. Ma`adamiyeh Al-sham, (1), 23.5.2002; (4), 28.6.2002; (7), 19.7.2002; (5), 30.8.2002; (15), 9.9.2002; (1), 4.12.2002; (1), 9.4.2003; (1), 19.5.2003; (7), 23.6.2003; (6), 6.7.2003; (25), 11.7.2003; (7), 23.7.2003; (6), 13.8.2003. Wadi AlQaren, (6), 10.7.2002; (2), 14.3.2002; (1), 19.2.2003. 
Yabrud, (7), 2.8.2001; (1), 26.9.2001; (1), 4.4.2002; (1), 26.6.2002; (8), 8.8.2002; (6), 11.9.2002. Kulaiaa, (2), 7.8.2002, Beet-jen Mazraa, (14), 7.8.2002; (11), 29.8.2002. Erneh, (5), 11.7.2002. Beqaasem, (1), 21.9.2001; (4), 11.7.2002; (1), 29.7.2002, Mugr Almeer, (2), 16.4.2003. Kafr- Hawar, (1), 7.8.2002. Zarzar, (1), 19.9.2001; (1), 28.11.2001; (4), 30.4.2003; (1), 14.6.2003. Deir Al-ashairr, (9), 17.10.2001; (3), 28.11.2001; (4), 11.6.2002; (4), 17.9.2002. Addimass, (1), 10.7.2002; (10), 5.9.2002; (3), 17.9.2002; (2), 30.4.2003. Al-Hameh, (2), 14.6.2003. Azzabadani, (14), 27.6.2002; (1), 14.6.2003. Rankaus, (6), 10.6.2003. Sarkha, (25), 26.6.2002; (6), 11.9.2002; (6), 21.5.2003. Seydnaya, (7), 2.8.2001; (4), 26.6.2002. Qatana, (1), 14.8.2003. Deir Atyeh, (4), 21.5.2003. Jeesrin, (2), 29.12.2002. Ashiphonieh, (1), 25.9.2002. Annashabyeh, (1), 28.8.2001. Damascus: Qasioun, (14), 13.5.2003. Al-Quneitra: Jubata AlKhashab, (2), 20.3.2002; (1), 28.2.2002; (2), 16.5.2002; (2), 26.8.2003; (3), 22.5.2004. Hadar, (2), 10.9.2001; (2), 31.7.2002; (1), 12.9.2002. Beer Ajam, (2), 12.9.2002; (1), 2.10.2002. As`Sweida: Al-Kafr, (1), 18.10.2001; (1), 29.11.2001; (2), 9.5.2002; (5), 6.6.2002; (1), 1.8.2002; (10), 23.10.2002; (5), 11.5.2003; (2), 12.6.2003. Shahba, (1), 13.3.2002; (1), 11.12.2002. Ein alarab, (1), 28.6.2002; (2), 4.7.2002; (2), 17.7.2002; (1), 10.9.2002. Orman Mountain, (3), 11.4.2002. Orman, (1), 30.5.2002; (3), 21.6.2002; (1), 4.7.2002; (1), 11.7.2002; (2), 17.7.2002. Ghoujygoujyat Mountain, (1), 10.9.2002, (1), 4.8.2003. Taamri Mountain, (2), 25.7.2003; (1), 8.8.2003. Qanawat, (1), 11.9.2001; (1), 13.3.2002; (4), 11.4.2002; (1), 9.5.2002; (1), 17.7.2002; (2), 1.8.2002. Dar`a: Shihab, (1), 25.7.2002. Wadi Shihab, (1), 13.5.2003. Zeizon, (1), 21.3.2002; (1), 28.3.2002; (4), 10.4.2002; (2), 25.4.2002; (10), 9.5.2002; (4), 29.5.2002. Nahaj, (6), 4.10.2001.

Remarks: This species has a wide range across Europe, North Africa to the Far East and North America (Kuznetsov, 1997). In Syria, it was recorded from the Golan Heights, Mount Hermon, Wadi Al Qaren, As`Sweida and northern Syria (Kabakibi, 1993; Halperin et al., 1995; Almatni et al., 1999; Ka'ada, 2002). Jalilvand et al. (2014) found that this species predates on Planococcus ficus in Iran.

Host species: Acer sp., Aloysia citrodora, Amygdalus sp., Biota orintalis, Cedrus libani, Citrus sp., Cupressus sp., Cydonia vulgaris, Elaeagnus angustifolia, Eriobotrya japonica, Eucalyptus camaldulensis, Faba vulgaris, Ficus carica, Glycyrrhiza glabra, Hibiscus esculentus, Inula viscosa, Juglans regia, Ligustrum sp., Medicago sativa, Morus alba, Nerium oleander, Olea europaea, Prunus armeniaca, Prunus vulgaris, Pinus sp., Pistacia vera, Populus sp., Prunus avium, Prunus mahaleb, Punica granatum, Pyrus communis, Quercus calliprinos, Robinia pseudoacacia, Rosa sp., Rubus idaeus, Salix sp., Solanum melongena., Ulmus campestris, Urtica sp., Vitis vinifera and Zea mays,

\section{Oenopia oncina (Olivier, 1808) (Figs. 5V and 6O)}

Material examined (6): Damascus Rural: Deir Alashairr, (1), 17.10.2001; (1), 11.6.2002. Bludan, (1), 14.6.2003. Rankus Mountain, (1), 21.5.2003, Rankaus, (1), 10.6.2003. Dar`a: Wadi Shihab, (1), 13.5.2003.

Remarks: This species is distributed in the Eastern Mediterranean extending to Central Asia (Iablokoff-
Khnzorian, 1982). This species represents a new record to Syria.

Host species: Amygdalus sp., Medicago sativa, Onobryclus sp. and Punica granatum.

\section{Propylea quatuordecimpunctata (Linnaeus, 1758) (Figs.} 5W and 6P)

Material examined (121): Damascus Rural: Qatana, (1), 5.7.2002. Ahmadia, (1), 25.9.2002. Assaida Zainab, (2), 11.9.2001; (3), 13.3.2002. Jeesrin, (3), 9.2.2003. Ashiphonieh, (1), 25.9.2002, Attall, (7), 3.9.2001; (2), 4.4.2002; (1), 8.5.2002; (1), 26.6.2002; (18), 10.7.2002; (6), 15.7.2005. Kharabo, (1), 22.8.2001; (1), 26.9.2001; (2), 25.10.2001; (1), 15.5.2002; (9), 23.5.2002; (7), 11.7.2002; (3), 24.7.2002; (1), 8.8.2002; (8), 25.9.2002; (13), 9.10.2002; (3), 2.4.2003; (10), 25.5.2003; (3), 2.7.2003; (3), 6.8.2003. Ma`adamiyeh Al-sham, (3), 28.6.2002; (3), 19.7.2002; (1), 19.5.2003; (1), 23.6.2003; (1), 11.7.2003; (1), 23.7.2003.

Remarks: This is a Trans-Palaearctic species with wide range of distribution through Europe, across Asia reaching Korea and Japan (Iablokoff-Khnzorian, 1982). Regionally, it was recorded from Lebanon and Palestine (Talhook, 1961; Halperin et al., 1995). Recorded by Al Jundi and Ahmad (1999) from the coastal regions, and from Damascus by Bascheer and Abo Alshamat (2004).

Host species: Cucumis sativus, Cucurbita pepo, Cydonia vulgaris, Faba vulgaris, Foeniculum vulgare Hordium vulgare, Juglans regia, Medicago sativa, Morus alba, Populus sp., Punica granatum, Solanum melongena, Triticum sp. Urtica sp., and Zea mays.

Psyllobora (Thea) vigintiduopunctata (Linnaeus, 1758) (Figs. 5X and 6Q)

Material examined (2): Damascus Rural: Attall, (2), 3.9.2001.

Remarks: This is a Palaearctic species with a wide distribution in Europe (Harde, 1999). Aslan and Uygun (2005) reported this species from Turkey on aphids. This species was listed by El Hariri (1968) from Syria. Recorded from northern Syria and the coastal regions by Ka'ada (2002) and Younes et al. (2015).

Host species: Vitis vinifera.

\section{Assocaition of coccinellids with their host plants}

The coccinellids in southern Syria are associated with 90 plant species, number of plants per species of coccinellid ranged between considered 1 and 45 (12.72 \pm 13.04, Average \pm STDEV). 29 coccinellid species were found on fewer than 10 plants, thus specialists; whereas seven species were found on more than 30 plant species and were considered generalists (Figure 7). Generalist species include (from highest to lowest number of plant hosts): Coccinella septempunctata, Oenopia conglobata, Coccinella undecimpunctata, Stethorus gilvifrons, Scymnus apetzi, Hippodamia variegata, and Scymnus flavicollis. 


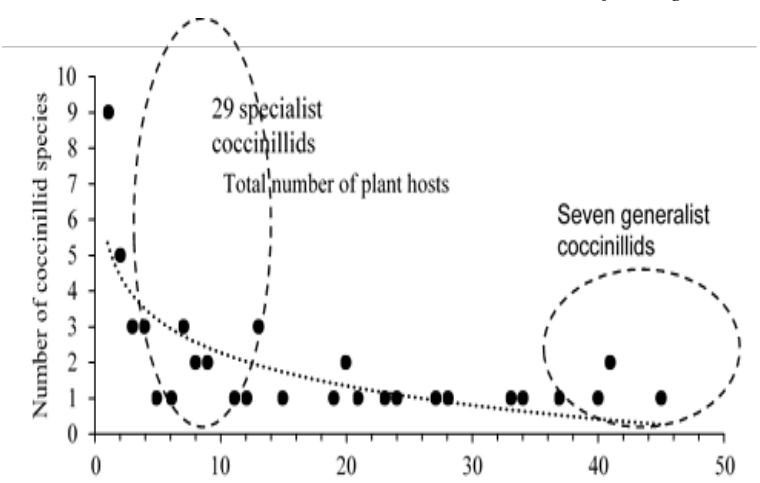

Figure 7. Occurrence of coccinellid species on plant hosts.

On the other hand, the number of coccinellid species per plant host ranged between 1 and 26 different species 7.07 \pm 6.87 (Average \pm STDEV). With 54 plant species $(60 \%$ of all plants) had $=<5$ different coccinillids, whereas, 18 plants (20\% of all plants) had 15-26 different coccinillids (Figure 8). These preferred plants include (from highest to lowest number of coccinillids): Punica granatum, Quercus calliprinos, Amygdalus sp., Citrus sp., Juglans regia, Morus alba, Zea mays, Pinus sp., Urtica sp., Medicago sativa, Ficus carica, Populus spp., Rubus idaeus, Malus communis, Pyrus communis, Cupressus sp., Prunus avium, and Prunus armeniaca. Additionally, at least 75 species of these plant hosts have agricultural value including Punica granatum, Amygdalus sp., Malus communis, Pyrus communis, Morus alba, and Citrus sp.

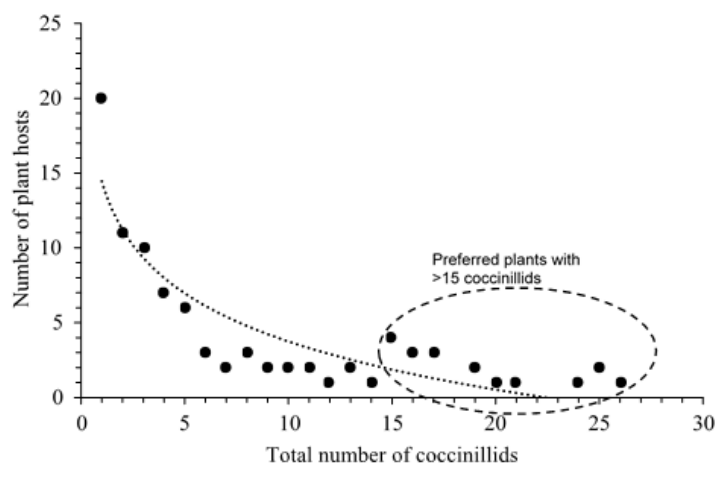

Figure 8. Diversity of coccinellid species on plant hosts.

\section{Discussion}

In this study, a total of 51 species were recorded. Fifteen are new records for Syria (Table 2). This high number of new records is not surprising, due to the paucity of systematic studies on the coccinellid fauna of Syria. Within the Middle East, Turkey enjoys the highest number of coccinellids reaching up to 84 species (Yurtsever, 2001), followed by Palestine with 71 species (Halperin et al., 1995; Najajrah et al., 2019). The number of coccinellids species of Syria is higher than those recorded from Jordan, Saudi Arabia, and the United Arab Emirates with species 16, 35, and 22 respectively (Fürsch, 1979; Allawi, 1989; aimundo and van Harten, 2000).

Stethorus gilvifrons and Hippodamia variegata were the most abundant species, and constituted $18.5 \%$ and $14 \%$ of the collected specimens respectively. The former species was very efficient predator, essentially feeding on mites. Aslan and Uygun (2005) reported this species from Turkey on aphids. Also, it feeds on all stages of the whitefly Bemisia tabaci (Al-Duhawi et al., 2006).
Based on species incidences among sites, three groups of species may be distinguished: there are 19 rare or "satellite" species which were recorded from less than 10 localities with low abundance; 5 modal species found at an intermediate number of sites over larger areas, and 13 ubiquitous "core", species recorded from more than 40 sites with 152-1368 specimens per species (Table 3). Five species (A. novemdecimpunctata, D. rubidus, $E$. octosignatus, $N$. caucasicus and $P$. setulosus) are considered as very rare species, since only one specimen of each was collected. Hippodamia variegata, Stethorus gilvifrons, Oenopia conglobata, Coccinella undecimpunctata agyptica, Coccinella septempunctata and Scymnus pharaonis respectively, are widely distributed in the study area.

More coccinellid species were collected in agricultural areas (47 species) than in non-agricultural areas (28 species). Only four specieces (E. undulatus, $E$. octosignatus, E. quadripustulatus and $S$. fraxini) were found in non-agricultural areas and were not found in agricultural areas.

Over ninety plant species were identified as hosts for these beetles; some plants have economic value, as fruit trees, crops, woods or ornamentals. The coccinellids on apple, citrus, almond, cherry and other fruit trees were identified. Since Syria is an agricultural country with a wide range of crops, the high diversity of coccinellids in Syria suggests the importance of these insects as biological control agents against harmful agricultural pests.

\section{Acknowledgements}

The authors are much indebted to Prof. Dr. Helmut Fürsch (Passau University, Germany) for his help to confirm the identification most species. We wish to thank Dr. Nashat Hamidan for his help in the preparation of figures.

\section{Disclosure statement}

No potential conflict of interest was reported by the authors.

\section{References}

Ahmadi AA and Yazdani A. 1993. The coccinellid fauna of Fars Province, part II: tribe Scymnini of subfamily Scymninae. JESI, 12/13: 1-22.

Al Jundi A and Ahmad M. 1999. [Contribution to the natural enemies for the most important citrus pests in the Coastal region of Syria]. Tishreen University for Research Studies, Agricultural Sciences Ser, 21: 29-40. [In Arabic].

Al-Duhawi SS, Ali AA and Sameer SH. 2006. The predation efficacy of the predators Stethorus gilvifrons (Muls) and Scolothrips sexmaculatus (Perg.) on Tobacco Whitefly Bemisia tabaci (Gen) which attack cotton plants. Arab J Plant Prot, 24: 112-117.

Alfieri A. 1976. The Coleoptera of Egypt. Memoires de la Societe entomologique d'Egypte, 5: 1-361.

Ali HA, Abdul-Rassoul MS and Swail MA. 1990. Systematic list of Coccinellidae recorded for Iraq. Bull Iraq Nat Hist Mus, 8: 4551.

Allawi TF. 1989. A list of predaceous coccinellids collected in Jordan. Dirasat, 16: 23-26. 
Almatni W and Khalil N. 2008. A primary survey of aphid species on almond and peach, and natural enemies of Brachycaudus amygdalinus in As-Sweida, Southern Syria. In: Ecofruit-13 ${ }^{\text {th }}$ International Conference on Cultivation Technique and Phytopathological Problems in Organic Fruit-Growing: Proceedings to the Conference from $18^{\text {th }}$ February to $20^{\text {th }}$ February 2008 at Weinsberg/Germany (pp. 109-115).

Almatni W, Mahmalji M and Al Rouz H. 1999. Recording and preliminary study of some natural enemies of the woolly apple aphid Eriosoma lanigerum (Hausmann) (Homoptera: Aphididae) in Syria. Toulouse, France, 39-44.

Aslan L. 2001. [Biological study of insect Planococcus citri (Risso) that strikes the citrus in the Syrian Coast]. Basel Al Asad Journal of Agricultural Sciences, 13: 9-31. [In Arabic].

Aslan MM and Uygun N. 2005. The aphidophagus Coccinellid (Coleoptera: Coccinellidae) species in Kahramanmaraş, Turkey. Turk J Zool., 29: 1-8.

Aswad N. 1998. [Biological Diversity of the Vascular Flora and the Arthropod Fauna in the Fornlok Forest that Represents Wet Forest Ecosystem and Prepared for Declarations as a Nature Reserve]. M. Sc. Theseis, Allepo University. 252 pp. [In Arabic].

Bascheer A and Abo Alshamat M. 2004. A preliminary survey of predators and parasitoid insects in Abo-Jarash regoin, Damascus. Bassel al-Assad Journal for Engineering Sciences- Agricultural, food, Chemical and Biotechnology. 19: 65- 85. [In Arabic].

Biranvand A, Nedvěd O, Tomaszewska W, Canepari C, Shakarami J, Fekrat L and Khormizi MZ. 2016. An annotated checklist of Microweiseinae and Sticholotidini of Iran (Coleoptera, Coccinellidae). ZooKeys, 587: 37-48.

Canepari C and Tedeschi M. 1977. Le sottospecie del Nephus quadrimaculatus (Herbst) (Coleoptera: Coccinellidae). Mem Soc Entomol Ital., 55: 101- 105.

Canepari C. 1983. Le specie italiane del gruppo Scymnus frontalis Fab. con descrizione di due nuove specie (Coleoptera: Coccinellidae). G Ital Entomol., 1: 179- 204.

Canepari C. 1991. Coleotteri Coccinellidi della provincia di Ferrara (Coleoptera: Coccinellidae). Quaderni della Stazione di Ecologia Museo Civico di Storia Naturale di Ferrara, 4: 43- 52.

Canepari C, Fürsch H. and Kreissl E. 1985. Die Hyperaspis-Arten von Mittel, West-und Sudeuropa. Systematik und verbreitung (Coleoptera: Coccinellidae). G Ital Entomol, 2: 223-252.

Chapin EA. 1965. The genera of the Chilocorini (Coleoptera: Coccinellidae). Bull Mus Comp Zool, 133: 227-271.

Chazeau J, Etienne J and Fürsch H. 1974. Les Coccinellidae de lîile de la Réunion (Insecta, Coleoptera). Bull Mus Natl Hist Nat 3 Ser., n. 210, Zoologie, 140: 265- 297.

Dauguet P. 1949. Les Coccinellini de France - L'entomologiste. Paris, 46pp.

Duverger C. 1991. Chilocorinae (Coleoptera: Coccinellidae) de France métropolitaine et de Corse. Bull Soc Linn Bordeaux, 19: 75- 95.

El-Hariri G. 1968. A List of Recorded Syrian Insects and Acari. Part 1. Faculty of Agriculture, University of Aleppo, Alleppo, 160 pp.

El-Hariri G. 1971. A List of Recorded Insect Fauna of Syria. Part 2. Faculty of Agriculture, University of Aleppo, Aleppo, 306 pp.

Fallahzadeh M, Abdimaleki R and Saghaei N. 2013. Contribution to the knowledge of the ladybird beetles (Coleoptera, Coccinellidae), predators of mealybugs (Hemiptera, Pseudococcidae) in Hormozgan Province, Southern Iran. Linz Biol Beitr, 45: 673-679.
Fürsch 1967a. Familie 62. Coccinellidae (Marienkäfer). In: Die Käfer Mittel-Europas. Freude H, Harde K and Lohse G. (Eds.). Band 7. pp. 227-278. Krefeld.

Fürsch 1967b. Scymnus (Sidis) hiekei Fürsch, als feind von Pseudococcus citri Risso. Entomophaga, 12: 309- 310.

Fürsch H and Uygun N. 1980. Neue Scymnini aus der Türkei. NachrBl Bayer Ent, 29: 109-118.

Fürsch H. 1965. Die palaearktischen Arten der Scymnusbipuctatus Gruppe und die europäischen Vertreter der Untergattung Sidis. Mitt Munch Entomol Ges, 55: 178-213.

Fürsch H. 1979. Insects of Saudi Arabia. Coleoptera: Fam. Coccinellidae. Fauna of Saudi Arabia, 1: 235-248.

Fürsch H. 1989. The Arabian species of the Scymnus (Pullus) guimeti-group (Coleoptera, Coccinellidae). Fauna of Saudi Arabia, 10: 113-123.

Fürsch H. 1994. Neue Coccinelliden-Art aus Israel (Coleoptera). Ann Hist-Nat Mus Natl Hung, 86: 43- 44.

Gourreau JM. 1974. Contribution a I Etude de la fauna de France: Systematique de la Tribu des Scymnini (Coccinellidea). Annnales de Zoologie, Ecologie Animale. INRA, Institut National De La Recherche Agronomique. Paris. 223 pp.

Halperin J, Merkl O and Kehat M. 1995. An annotated list of the coccinellidae (coleoptera) of Israel and adjacent areas. Phytoparasitica, 23: 127-137.

Harde KW. 1999. A Field Guide in Coulour to Beetles. Blitz editions. Prague. 333 pp.

Hodek I and Honek A. 1996: Ecology of Coccinellidae. Kluwer Academic Publishers. The Netherlands. 464 pp.

Hodek I. 1973. Biology of Coccinellidae. Dr. W. Junk N.V., Pub., The Hage, 260 pp.

Honek A. 2015. Distribution and habitats, In: Ecology and Behaviour of the Ladybird Beetles (Coccinellidae). Hodek I., van Emden, H.F. and Honek, A. (Eds.). Blackwell Publishing Ltd, pp. 110-140.

Iablokoff-Khnzorian SM. 1971. Synopsis des Hyperaspis Paléartiqes (Col.: Coccinellidae). Ann Soc Entomol Fr (N. S.), 7: 163- 200.

Iablokoff-Khnzorian SM. 1982. Les Coccinelles, CoélèopteresCoccinellidae, Tribu Coccinellini des regions Paléartique et Orientale. Société nouvelles des éditions boubée 11, place SaintMichel. Paris, 568 pp.

Jalilvand Kh, Shirazi M, Fallahzadeh M, Vahedi HA, Samih MA and Moeini Naghadeh N. 2014. Survey of natural enemies of mealybug species (Hemiptera, Pseudococcidae) in Kermanshah province, Western Iran to inform biological control research. $J$ Entomol Res Soc, 16: 1-10.

Ka'ada F. 2002. [Effect of Different Insecticeds in Cotton and Their Natural Enemies of Predacious Insects and Parasites in Cotton Fields in Northern Syria]. Ph. D. thesis, Allepo University. 196 pp. [in Arabic].

Kabakibi MM. 1993. [Animal biodiversity in a Mediterranean forest ecosystem-Wadi Al Qaren Nature Reserve (West of Damascus)]. $33^{\text {rd }}$ Scientific Week. [In Arabic].

Klausnitzer B and Klausnitzer H. 1986. Marienkäfer (Coccinellidae) 3 Überarb. Aufl. Wittenberg: A. Ziemsen Verlag, 104 pp.

Kováŕ I. 2007. Family Coccinellidae Latreille 1807. Pp. 568-631. In: Catalogue of Palaearctic Coleoptera. Löbl I. and Smetana A. (eds.). Vol. 4. Elateroidea, Derodontoidea, Bostrichoidea, Lymexyloidea, Cleroidea, Cucujoidea. Apollo Books, Stenstrup, 935 pp. 
Kuznetsov VN. 1997. Lady Beetles of the Russian Far East. Memoir No. 1, Center for Systematic Entomology, The Sandhill Crane Press Inc., Gainesville, 248 pp.

Lababidi MS and Zebitz CP. 1995. Preliminary study on the pistachio psyllid (Agonoscena targionii Lich.) (Psyllidae: Homoptera) and its associated natural enemies in some regions of Syria. Arab J Plant Prot, 13: 62- 68. [In Arabic].

Le Monnier Y and Livory A. 2003. Une enquête MancheNature: Atlas des Coccinelles de la Manche. Les Dossiers de Manche-Nature $\mathrm{N}^{\circ}$ 5, 206 p.

Leeper JR. 1975. A Review of the Hawaiian Coccinellidae. Technical Report No. 53, Hawaii national park library, Honolulu, Hawaii, 54 pp.

Majerus MEN and Kearns P. 1989. Ladybirds. No. 10 Naturalists` Handbooks Series. Richmond Publishing Co. Ltd. Slough, England, 103pp.

Mesbah RA, Nozari J, Allahyari H and Khormizi MZ. 2016. Checklist and distribution of lady beetles (Coleoptera: Coccinellidae) in Iran. Iran J Anim Biosyst, 12: 1-35.

Moodi S. 1994. The Coccinellids (Col.: Coccinellidae) Fauna of South East Khorasan, Iran. M.Sc. Thesis, Shahid Chamran University, Ahvaz, Iran, 158p. [In Persian].

Najajrah MH, Swaileh KM and Qumsiyeh MB. 2019. Systematic list, geographic distribution and ecological significance of lady beetles (Coleoptera: Coccinellidae) from the West Bank (Central Palestine). Zootaxa, 4664: 1-46.

Özden Ö, Uygun N and Kersting U. 2006. Ladybird beetles (Coccinellidae: Coleoptera) from northern Cyprus, including six new records. Zool Middle East, 39: 97- 100.

Poorani J. 2004. An annotated checklist of the Coccinellidae of the Indian region. World Wide Web electronic publication. www.angelfire.com/bug2/j.checklist.pdf. [accessed on 15.3.2010].

Raimundo AAC, Alves MLG. 1986. Revisăo dos Coccinelideos de Portugal. Universidade de Évora, Évora, Évora, 103 pp.
Raimundo AAC and van Harten A. 2000. An annotated checklist of the Coccinellidae (Insecta: Coleoptera) of Yemen. Fauna of Arabia, 18: 211-243.

Raimundo AC, Fürsch H and van Harten A. 2008. Order Coleoptera, family Coccinellidae. In: Arthropod Fauna of the United Arab Emirates. Van Harten A. (Ed.). Dar Al Ummah printing. Adu Dhabi, United Arab Emirates, 1: 217-239.

Seago AE, Giorgi JA, Li J and Ślipiński A. 2011. Phylogeny, classification and evolution of ladybird beetles (Coleoptera: Coccinellidae) based on simultaneous analysis of molecular and morphological data. Mol Phylogenet Evol, 60: 137-151.

Ślipiński A. 2007. Australian Ladybird Beetles (Coleoptera: Coccinellidae) their Biology and Classification. Australian Biological Resources Study, Canberra, 286 pp.

Talhook AS. 1961. Records of entomophagous insects from Lebanon. Entomophaga, 6: 207-209.

Uygun N and Fürsch H. 1981. Die Hyperaspis-Arten der Türkei (Coleoptera, Coccinellidea). NachrBl Bayer Ent, 30: 12- 16.

Uygun N. 1981. Türkiye Coccinellidea (Coleoptera) faunasi üzerinde taksonomik araştirmalar. Dilek Matbaasi, Adana, 111 pp. [In Turkish].

Winkler A. 1927. Catalogus Coleopterorum Regionis Palaearcticae. A. Winkler, Wien, 1698 pp.

Younes GH, Ahmad M and Ali N. 2015. Morphological, biological and ecological studies of the mycophagous ladybird Psyllobora vigintiduopunctata L. (Coleoptera: Coccinellidae) on powdery mildew fungi in the coastal region of Syria. JJAS, 11: 483-494.

Yurtsever S. 2001. A Preliminary study on the Ladybirds (Coleoptera: Coccinellidae) of Edirne in North-Western Turkey. Turk J Zool, 25: 71-75.

Zhou X, Honek A, Powell W and Carter N. 1995. Variations in body length, weight, fat content and survival in Coccinella septempunctata at different hibernation sites. Entomol Exp Appl, 75: 99-107. 